\title{
Electromagnetic design of superconducting multipole corrector magnets based on sector coils
}

\author{
Alexandre Louzguiti ${ }^{*}$ " and Daniel Schoerling \\ CERN, CH-1211 Geneva 23, Switzerland
}

(Received 9 October 2019; published 28 January 2020)

\begin{abstract}
We present here analytical formulas derived for sector coils with $2 \mathrm{~N}$ poles, an iron screen and optional iron poles. These formulas can be used to produce optimized conceptual electromagnetic designs of superconducting cosine-theta corrector magnets with $2 \mathrm{~N}$ poles. The main guidelines of the corresponding algorithm, i.e., design algorithm for sextupoles and higher, which has been used to produce valid conceptual electromagnetic designs of the Future Circular Collider sextupole and octupole magnets, are also presented. Generic conceptual design studies performed with DASH and DASH2in1 (for nested corrector magnets configurations) are also shown and their outcome is discussed.
\end{abstract}

DOI: $10.1103 /$ PhysRevAccelBeams.23.012402

\section{INTRODUCTION}

During the design phase of particle accelerators, such as the High-Energy Large Hadron Collider (HE-LHC) or the hadron-hadron Future Circular Collider (FCC-hh) [1], the functional specifications of lattice or spool piece corrector magnets (sextupoles, octupoles, decapoles, and dodecapoles) are evolving alongside the accelerator design. The parameters of these corrector magnets are driven mainly by the requirements of linear and nonlinear [2] beam dynamics to allow for correction of multipole errors and effects from the insertion regions. Many design iterations with large variations in the required field strength and the integrated gradient are typically required. In order to explore quickly the possible parameter space we developed the design algorithm for sextupoles and higher (DASH). This algorithm, based on analytical formulas, allows to calculate a wide parameter range for superconducting cost efficient multipole magnets including the iron yoke, and if desired, iron poles. As the achievable magnetic field in multipole magnets decreases with increasing pole number, the field contributed by the iron is important and cannot be neglected as often done as a first approximation for high-field dipoles and quadrupoles [3].

The derived formulas have been benchmarked with ROXIE on configurations representative of LHC corrector magnets, and on specific electromagnetic designs established for the FCC-hh. The results always showed an agreement within the $5 \%$ range.

"alexandre.mehdi.louzguiti@cern.ch

Published by the American Physical Society under the terms of the Creative Commons Attribution 4.0 International license. Further distribution of this work must maintain attribution to the author(s) and the published article's title, journal citation, and DOI.
The sector coil geometry has been selected for the derivation of the formulas, as this geometry simplifies largely the required equations and is for multipole magnets very close to the geometry of the real magnet. Figure 1 shows as an example a sextupole magnet with all features implemented in the here presented algorithm DASH. In addition, in this paper comprehensive analytical formulas of the magnet strength and peak field on conductor in sector coils with $2 \mathrm{~N}$ poles and with an iron screen and optional iron poles are derived. These analytical expressions are complemented with equations allowing setting a specific margin along the magnet load line and taking into account magnet protection in a heuristic way, so that protection of the magnets without active individual extraction resistors remains possible.

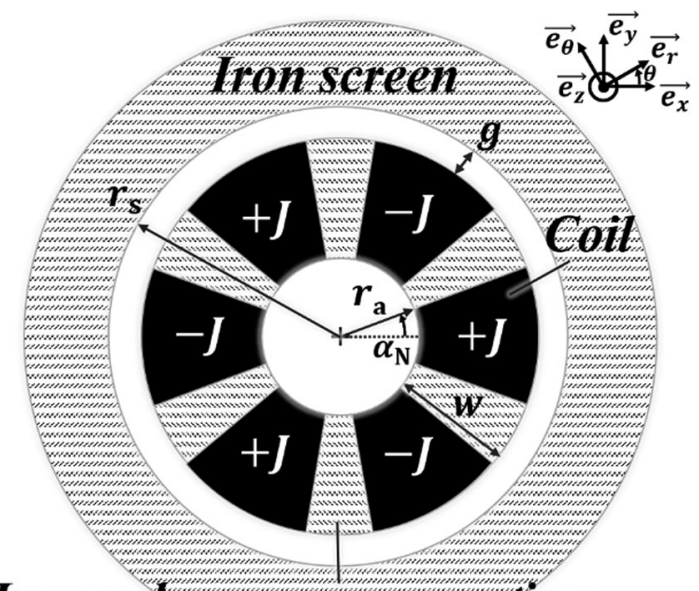

\section{Iron pole ornon-magnetic spacer}

FIG. 1. Sketch of a $2 N$-poles sector coil with iron screen and optional iron poles for $N=3$ (i.e., sextupole). 
Furthermore, the here presented analytical formulas of the magnetic vector potential, of the magnet strength and of the peak field on conductor in sector coils with $2 \mathrm{~N}$ poles, with iron screen and optional iron poles allow, contrary to published equations for sector coils [4-7], the following.

(i) First, they can be used for sector coils with any number of poles while the previous formulas were stopping at the octupole level. This allows to model decapoles and dodecapoles, as these magnets are used in present particle accelerators (e.g., LHC), and are also required for future ones (e.g., HE-LHC, FCC-hh). The general formulation also eases the implementation of these formulas in an algorithm.

(ii) Second, they take into account the partial saturation of the iron screen. They thus bring the analytical evaluation closer to the results of numerical models (e.g., ROXIE [7]).

(iii) Third, they are also able to take into account the magnetic effect of saturated iron poles in an accurate way.

\section{VECTOR POTENTIAL IN A SECTOR COIL}

In order to derive the magnetic vector potential generated in a sector coil, we have used a methodology that differs from the ones adopted in previously presented approaches. Indeed, in [4] Asner used the Biot-Savart law to express the magnetic field created by a field line and integrated this expression over the sector coil winding to obtain the magnetic field generated by the sector coil. In addition, he considered an unsaturated (i.e., infinite $\mu_{r}$ ) magnetic screen around the sector coil winding.

\section{A. Vector potential generated in a sector coil with iron screen and without iron poles}

Alternatively, we have first considered an infinite iron screen with permeability $\mu_{r}$ and have used the Laplace equation for the axial component $A_{z}$ of the magnetic vector potential, i.e., $\Delta A_{z}=0$, whose solution for a thin $K_{n} \cos (n \theta)$ surface current located at $r=r^{\prime}$ is, in polar coordinates $(r, \theta)$,

$$
A_{z}^{(k)}(r, \theta)=r_{s} \sum_{i=-\infty}^{+\infty}\left(\frac{r}{r_{s}}\right)^{i} \beta_{i}^{(k)}\left(r^{\prime}\right) \cos (i \theta),
$$

where $r_{s}$ is the internal radius of the iron screen as shown in Fig. 1. The index $k=1,2,3$ refers respectively to the zones inside the thin current shell (i.e., $r \leq r^{\prime}$ ), outside the thin current shell but inside the iron screen (i.e., $r^{\prime} \leq r \leq r_{s}$ ), and in the iron screen (i.e., $r \geq r_{s}$ ). The $\beta_{i}^{(k)}$ are coefficients depending on $r^{\prime}$; to compute them, we have combined the following magnetic field interface conditions at the boundaries between the three zones

$$
\begin{aligned}
& B_{r}^{(2)}\left(r^{\prime}, \theta\right)=B_{r}^{(1)}\left(r^{\prime}, \theta\right) \\
& B_{\theta}^{(2)}\left(r^{\prime}, \theta\right)=B_{\theta}^{(1)}\left(r^{\prime}, \theta\right)+\mu_{0} K_{n} \cos (n \theta) \\
& B_{r}^{(3)}\left(r_{s}, \theta\right)=B_{r}^{(1)}\left(r_{s}, \theta\right) \\
& B_{\theta}^{(3)}\left(r_{s}, \theta\right)=\mu_{r} B_{\theta}^{(2)}\left(r_{s}, \theta\right)
\end{aligned}
$$

as well as the equation linking the magnetic field to the vector potential, i.e., $\vec{B}=\vec{\nabla} \times \vec{A}$, and Eq. (1). Following this approach, we have been able to derive the following formulas for the magnetic vector potential in zones 1 and 2:

$$
\begin{aligned}
& A_{z}^{(1)}\left(r, \theta, r^{\prime}\right)=\frac{\mu_{0} K_{n}}{2 n} \cos (n \theta) r^{n}\left[r^{\prime 1-n}+a_{\mu} r_{\mathrm{s}}^{-2 n} r^{\prime n+1}\right], \\
& A_{z}^{(2)}\left(r, \theta, r^{\prime}\right)=\frac{\mu_{0} K_{n}}{2 n} \cos (n \theta)\left[r^{-n}+a_{\mu} r_{\mathrm{s}}^{-2 n} r^{n}\right] r^{\prime n+1},
\end{aligned}
$$

where $K_{n}$ is the amplitude of the thin $\cos (n \theta)$ surface current and

$$
a_{\mu}=\left(\mu_{r}-1\right) /\left(\mu_{r}+1\right) .
$$

Second, we have extended these formulas to the case of a thick $J_{n}\left(r^{\prime}\right) \cos (n \theta)$ current, considering a dependence of its amplitude $J_{n}$ on the radial position $r^{\prime}$ with $r_{a} \leq r^{\prime} \leq r_{a}+w$, where $r_{a}$ is the aperture radius of the sector coil and $w$ is its width as shown in Fig. 1. Using the fact that $K_{n}=J_{n}\left(r^{\prime}\right) d r^{\prime}$, this intermediate step in our calculation of the sector coil vector potential has led us to the following expressions of $A_{z}^{(a)}$ in the aperture (i.e., $r \leq r_{a}$ ) and of $A_{z}^{(b)}$ in the sector coil winding (i.e., $\left.r_{a} \leq r \leq r_{a}+w\right)$ :

$$
\begin{aligned}
A_{z}^{(a)}(r, \theta)= & \frac{\mu_{0}}{2 n} \cos (n \theta)\left[r^{n}\left(\int_{r^{\prime}=r_{a}}^{r_{a}+w} J_{n}\left(r^{\prime}\right) r^{\prime 1-n} d r^{\prime}\right)\right. \\
& \left.+a_{\mu} r_{s}^{-2 n} r^{n}\left(\int_{r^{\prime}=r_{a}}^{r_{a}+w} J_{n}\left(r^{\prime}\right) r^{\prime n+1} d r^{\prime}\right)\right] \\
A_{z}^{(b)}(r, \theta)= & \frac{\mu_{0}}{2 n} \cos (n \theta)\left[r^{-n}\left(\int_{r^{\prime}=r_{a}}^{r} J_{n}\left(r^{\prime}\right) r^{\prime n+1} d r^{\prime}\right)\right. \\
& +r^{n}\left(\int_{r^{\prime}=r}^{r_{a}+w} J_{n}\left(r^{\prime}\right) r^{\prime 1-n} d r^{\prime}\right) \\
& \left.+a_{\mu} r_{s}^{-2 n} r^{n}\left(\int_{r^{\prime}=r_{a}}^{r_{a}+w} J_{n}\left(r^{\prime}\right) r^{\prime n+1} d r^{\prime}\right)\right] .
\end{aligned}
$$

Third, the current distribution in a sector coil with $2 \mathrm{~N}$ poles (see Fig. 1) is given for $r_{a} \leq r^{\prime} \leq r_{a}+w$ by 
$J_{\mathrm{sec}}\left(r^{\prime}, \theta\right)= \begin{cases}(-1)^{k-1} J & \text { if } \theta \in \cup_{1 \leq k \leq 2 N}\left[\varphi_{k}-\alpha_{N} ; \varphi_{k}+\alpha_{N}\right] \\ 0 & \text { otherwise }\end{cases}$

where $J$ is the engineering current density in each sector, $N$ is half the number of poles, $\varphi_{k}=\pi(k-1) / N$ and

$$
\alpha_{N}=\pi /(3 N)
$$

is half the angular thickness of a sector, as shown in Fig. 1.

Since the $J_{\text {sec }}\left(r^{\prime}, \theta\right)$ distribution is a $2 \pi$-periodic function of $\theta$, it can be decomposed as a Fourier series, and, using the fact that it is symmetric with respect to the $x$ axis (see Fig. 1), we can directly express it as

$$
J_{\mathrm{sec}}\left(r^{\prime}, \theta\right)=\sum_{n=1}^{\infty} J_{n} \cos (n \theta)
$$

where $J_{n}$ are the harmonics given for $n \in \mathbb{N}^{*}$ by

$$
J_{n}=\frac{1}{\pi} \int_{\theta=0}^{2 \pi} J_{\mathrm{sec}}\left(r^{\prime}, \theta\right) \cos (n \theta) d \theta
$$

which is equivalent to

$$
J_{n}=\frac{2 J}{n \pi} \sin \left(n \alpha_{N}\right) \sum_{k=0}^{2 N-1} e^{i k \pi\left(1+\frac{n}{N}\right)}
$$

where $i$ is the imaginary unit.

Finally, we have summed the $A_{z}^{(a)}$ contributions given by Eq. (5) for each harmonic $J_{n}$ given by Eq. (10) from $n=1$ to infinity to obtain the following total magnetic vector potential in the aperture of a sector coil:

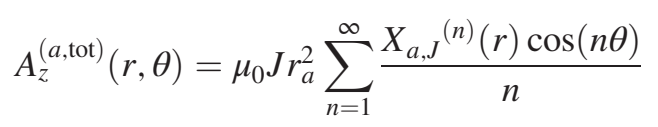

with $X_{a, J}^{(n)}(r)$ a dimensionless function of $r$ given by

$$
\begin{aligned}
X_{a, J}^{(n)}(r)= & \sum_{k=0}^{2 N-1} e^{i k \pi\left(1+\frac{n}{N}\right)} \frac{\sin \left(n \alpha_{N}\right)}{n \pi}\left(\frac{r}{r_{a}}\right)^{n} \\
& \times\left\{\frac{1}{n-2}\left[1-\left(\frac{r_{a}}{r_{a}+w}\right)^{n-2}\right]\right. \\
& +\frac{a_{\mu}}{n+2}\left(\frac{r_{a}+w}{r_{a}}\right)^{2}\left(\frac{r_{a}}{r_{s}}\right)^{n}\left(\frac{r_{a}+w}{r_{s}}\right)^{n} \\
& \left.\times\left[1-\left(\frac{r_{a}}{r_{a}+w}\right)^{n+2}\right]\right\},
\end{aligned}
$$

where the term

$$
\frac{1}{n-2}\left[1-\left(\frac{r_{a}}{r_{a}+w}\right)^{n-2}\right]
$$

has to be replaced by $\ln \left(1+w / r_{a}\right)$ if $n=2$. Similarly, we have summed the $A_{z}^{(b)}$ contributions given by Eq. (5) for each harmonic $J_{n}$ given by Eq. (10) from $n=1$ to infinity to obtain the following total magnetic vector potential in the winding of a sector coil:

$$
\begin{aligned}
& A_{z}^{(b, \text { tot })}(r, \theta) \\
& =\mu_{0} J r_{a}{ }^{2} \sum_{n=1}^{\infty} \frac{\left[X_{b, J}^{(n)}(r)+Y_{b, J}^{(n)}(r)+Z_{b, J}^{(n)}(r)\right] \cos (n \theta)}{n}
\end{aligned}
$$

with $\quad X_{b, J}^{(n)}(r), Y_{b, J}^{(n)}(r), Z_{b, J}^{(n)}(r) \quad$ dimensionless functions of $r$ given by

$$
\begin{aligned}
X_{b, J}^{(n)}(r) & =\frac{\sin \left(n \alpha_{N}\right)}{\pi n(n+2)} \sum_{k=0}^{2 N-1} e^{i k \pi\left(1+\frac{n}{N}\right)}\left(\frac{r}{r_{a}}\right)^{2}\left[1-\left(\frac{r_{a}}{r}\right)^{n+2}\right], \\
Y_{b, J}{ }^{(n)}(r) & =\frac{\sin \left(n \alpha_{N}\right)}{\pi n(n-2)} \sum_{k=0}^{2 N-1} e^{i k \pi\left(1+\frac{n}{N}\right)}\left(\frac{r}{r_{a}}\right)^{2}\left[1-\left(\frac{r}{r_{a}+w}\right)^{n-2}\right], \\
Z_{b, J}{ }^{(n)}(r) & =\frac{\sin \left(n \alpha_{N}\right)}{\pi n(n+2)} \sum_{k=0}^{2 N-1} e^{i k \pi\left(1+\frac{n}{N}\right)} a_{\mu}\left(\frac{r_{a} r}{r_{s}^{2}}\right)^{n}\left[\left(1+\frac{w}{r_{a}}\right)^{n+2}-1\right] . \\
\text { If } n & =2 \text {, replace } \frac{1-\left[r /\left(r_{a}+w\right)\right]^{n-2}}{n-2} \text { by } \ln \left(\frac{r_{a}+w}{r}\right) \text { in } Y_{b, J}{ }^{(n)}(r) .
\end{aligned}
$$

To assess the validity of the vector potential formulas obtained in Eqs. (11) to (14), we have derived the magnetic field associated with it using $\vec{B}=\vec{\nabla} \times \vec{A}$ (see the Appendix) and have compared it in [8] to the magnetic field expressions in the aperture and in the winding of a sector coil given by Asner in [4] for the case $a_{\mu}=1$ [see Eq. (4) above], i.e., considering the iron screen as unsaturated [see discussion on Eq. (31) below]. The obtained 
expressions were in exact analytical agreement with the ones presented in [4].

\section{B. Contribution of saturated iron poles to the vector potential generated in a sector coil}

In the presence of iron poles, under the assumptions that they are fully saturated (i.e., magnetic field larger than about $2.5 \mathrm{~T}$ in their volume) and that their magnetization is purely radial (see Fig. 2), it is possible to take into account their contribution in the magnetic vector potential analytically. Indeed, these two assumptions, which are usually fulfilled under nominal operating conditions of superconducting corrector magnets (e.g., LHC MS, see [8]), have enabled us to express the iron poles magnetization as $\vec{M}= \pm M_{\text {sat }} \vec{e}_{r}$, where $M_{\text {sat }}$ is the saturation magnetization of the iron poles (e.g., $M_{\text {sat }}=1.7 \times 10^{6} \mathrm{~A} / \mathrm{m}$ for standard iron). This means that the magnetization distribution in a sector coil with saturated iron poles (see Fig. 2) is given for $r_{a} \leq r^{\prime} \leq r_{a}+w$ by

$M_{\mathrm{sec}}\left(r^{\prime}, \theta\right)= \begin{cases}(-1)^{k} M_{\mathrm{sat}} & \text { if } \theta \in \mathrm{\cup}_{1 \leq k \leq 2 N}\left[\varphi_{k}+\alpha_{N} ; \varphi_{k+1}-\alpha_{N}\right] \\ 0 & \text { otherwise }\end{cases}$

where $\varphi_{k}=\pi(k-1) / N$ and $\alpha_{N}$ is given by Eq. (7). Since the $M_{\mathrm{sec}}\left(r^{\prime}, \theta\right)$ distribution is a $2 \pi$-periodic function of $\theta$, it can be decomposed as a Fourier series, and, using the fact that it is antisymmetric with respect to the $x$ axis (see Fig. 2), we can directly express it as

$$
M_{\mathrm{sec}}\left(r^{\prime}, \theta\right)=\sum_{n=1}^{\infty} M_{n} \sin (n \theta),
$$

where $M_{n}$ are the harmonics given for $n \in \mathbb{N}^{*}$ by

$$
M_{n}=\frac{1}{\pi} \int_{\theta=0}^{2 \pi} M_{\mathrm{sec}}\left(r^{\prime}, \theta\right) \sin (n \theta) d \theta
$$

which is equivalent to

$$
M_{n}=-\frac{2 M_{\text {sat }}}{n \pi} \sin \left(\frac{n \alpha_{N}}{2}\right) \sin \left(\frac{3 n \alpha_{N}}{2}\right) \sum_{k=0}^{2 N-1} e^{i k \pi\left(1+\frac{n}{N}\right)} \text {, }
$$

where $i$ is the imaginary unit. We have then converted the magnetization distribution $M_{\mathrm{sec}}\left(r^{\prime}, \theta\right)$ into an equivalent current distribution $J_{\mathrm{sec}}^{M}\left(r^{\prime}, \theta\right)$ using the equation $\overrightarrow{J^{M}}=\vec{\nabla} \times \vec{M}$; this has led us to

$$
J_{\mathrm{sec}}^{M}\left(r^{\prime}, \theta\right)=-\frac{1}{r^{\prime}} \frac{\partial M_{\mathrm{sec}}}{\partial \theta}\left(r^{\prime}, \theta\right)
$$

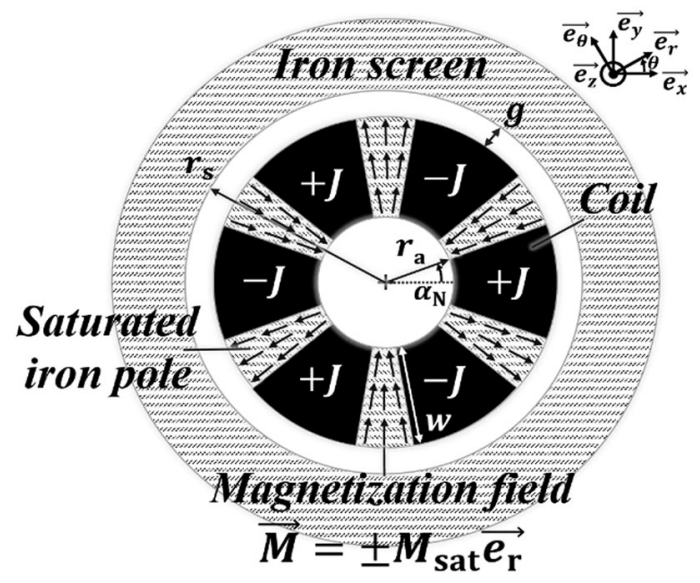

FIG. 2. Sketch of a $2 N$-poles sector coil with iron screen, saturated iron poles and associated magnetization field for $N=3$ (i.e., sextupole).

Using Eqs. (16), (18) and (19), we can finally express $J_{\mathrm{sec}}^{M}\left(r^{\prime}, \theta\right)$ as

$$
J_{\mathrm{sec}}^{M}\left(r^{\prime}, \theta\right)=\sum_{n=1}^{\infty} J_{n}^{M}\left(r^{\prime}\right) \cos (n \theta),
$$

where $J_{n}^{M}\left(r^{\prime}\right)$ are the harmonics given for $n \in \mathbb{N}^{*}$ by

$J_{n}^{M}\left(r^{\prime}\right)=\frac{2 M_{\mathrm{sat}}}{\pi r^{\prime}} \sin \left(\frac{n \alpha_{N}}{2}\right) \sin \left(\frac{3 n \alpha_{N}}{2}\right) \sum_{k=0}^{2 N-1} e^{i k \pi\left(1+\frac{n}{N}\right)}$

From this result, it has then been straightforward to calculate the magnetic contribution of the saturated iron poles in the aperture and in the winding of a sector coil following the methodology described in Sec. II A. Indeed, we have again summed the $A_{z}^{(a)}$ contributions given by Eq. (5) for each harmonic $J_{n}^{M}\left(r^{\prime}\right)$ given by Eq. (21) from $n=1$ to infinity to obtain the following total magnetic vector potential in the aperture due to the saturated iron poles of a sector coil:

$$
A_{z}^{(a, \text { tot })}(r, \theta)=\mu_{0} M_{\text {sat }} r_{a} \sum_{n=1}^{\infty} \frac{X_{a, M}^{(n)}(r) \cos (n \theta)}{n}
$$

with $X_{a, M}{ }^{(n)}(r)$ a dimensionless function of $r$ given by

$$
\begin{aligned}
X_{a, M}^{(n)}(r)= & \frac{1}{\pi} \sin \left(\frac{n \alpha_{N}}{2}\right) \sin \left(\frac{3 n \alpha_{N}}{2}\right)\left(\frac{r}{r_{a}}\right)^{n} \\
& \times\left\{\frac{1}{n-1}\left[1-\left(\frac{r_{a}}{r_{a}+w}\right)^{n-1}\right]\right. \\
& +\frac{a_{\mu}}{n+1}\left(\frac{r_{a}+w}{r_{a}}\right)\left(\frac{r_{a}}{r_{s}}\right)^{n}\left(\frac{r_{a}+w}{r_{s}}\right)^{n} \\
& \left.\times\left[1-\left(\frac{r_{a}}{r_{a}+w}\right)^{n+1}\right]\right\} \sum_{k=0}^{2 N-1} e^{i k \pi\left(1+\frac{n}{N}\right)},
\end{aligned}
$$


where the term

$$
\begin{aligned}
& A_{z}^{(b, \text { tot })}(r, \theta) \\
& \quad=\mu_{0} M_{\text {sat }} r_{a} \sum_{n=1}^{\infty} \frac{\left[X_{b, M}^{(n)}(r)+Y_{b, M}{ }^{(n)}(r)+Z_{b, M}{ }^{(n)}(r)\right] \cos (n \theta)}{n}
\end{aligned}
$$

has to be replaced by $\ln \left(1+w / r_{a}\right)$ if $n=1$.

Using the same approach, but summing the $A_{z}^{(b)}$ contributions given by Eq. (5) instead, the total magnetic vector potential in the winding due to the saturated iron poles is

with $X_{b, M}{ }^{(n)}(r), Y_{b, M}{ }^{(n)}(r), Z_{b, M}{ }^{(n)}$ dimensionless functions of $r$ given by

$$
\begin{aligned}
X_{b, M}{ }^{(n)}(r) & =\frac{\sin \left(\frac{n \alpha_{N}}{2}\right) \sin \left(\frac{3 n \alpha_{N}}{2}\right)}{\pi(n+1)} \sum_{k=0}^{2 N-1} e^{i k \pi\left(1+\frac{n}{N}\right)} \frac{r}{r_{a}}\left[1-\left(\frac{r_{a}}{r}\right)^{n+1}\right], \\
Y_{b, M}{ }^{(n)}(r) & =\frac{\sin \left(\frac{n \alpha_{N}}{2}\right) \sin \left(\frac{3 n \alpha_{N}}{2}\right)}{\pi(n-1)} \sum_{k=0}^{2 N-1} e^{i k \pi\left(1+\frac{n}{N}\right)} \frac{r}{r_{a}}\left[1-\left(\frac{r}{r_{a}+w}\right)^{n-1}\right], \\
Z_{b, M}{ }^{(n)}(r) & =\frac{\sin \left(\frac{n \alpha_{N}}{2}\right) \sin \left(\frac{3 n \alpha_{N}}{2}\right)}{\pi(n+1)} \sum_{k=0}^{2 N-1} e^{i k \pi\left(1+\frac{n}{N}\right)} a_{\mu}\left(\frac{r_{a} r}{r_{s}^{2}}\right)^{n}\left[\left(1+\frac{w}{r_{a}}\right)^{n+1}-1\right] \\
\text { If } n & =1 \text {, replace } \frac{1-\left[r /\left(r_{a}+w\right)\right]^{n-1}}{n-1} \text { by } \ln \left(\frac{r_{a}+w}{r}\right) \text { in } Y_{b, M}{ }^{(n)}(r) .
\end{aligned}
$$

\section{MAGNET STRENGTH AND PEAK FIELD ON CONDUCTOR IN A SECTOR COIL}

From the analytical formulas of the vector potential in a sector coil, expressions of the magnet strength and peak field on conductor are derived here. These equations are intended to provide analytical tools to the magnet designer to rapidly and trustworthily evaluate the magnet performance and stability.

\section{A. Magnet strength}

The classical expression of the magnetic vector potential inside the aperture [5-7] is

$$
A_{z}^{(a \text {,tot })}(r, \theta)=\sum_{n=1}^{\infty} \frac{r^{n}}{r_{0}{ }^{n-1}} \frac{A_{n}\left(r_{0}\right) \sin (n \theta)-B_{n}\left(r_{0}\right) \cos (n \theta)}{n},
$$

where $r_{0}$ is the reference radius. Using this expression, Eqs. (11) and (22), and the fact that $\cos (n \theta)_{n \in \mathbb{N}^{*}}$ and $\sin (n \theta)_{n \in \mathbb{N}^{*}}$ are linearly independent families, we can deduce that, for $n \in \mathbb{N}^{*}$

$$
\begin{aligned}
& A_{n}\left(r_{0}\right)=0, \\
& B_{n}\left(r_{0}\right)=-\mu_{0} r_{a} \frac{r_{0}{ }^{n-1}}{r^{n}}\left[J r_{a} X_{a, J}^{(n)}(r)+M_{\mathrm{sat}} X_{a, M}^{(n)}(r)\right] .
\end{aligned}
$$

Since the magnet strength $S$-which corresponds to the magnetic field in $\mathrm{T}$ for a dipole, the gradient in $\mathrm{T} / \mathrm{m}$ for a quadrupole and so on for higher order magnets - is defined as

$$
S=\frac{B_{N}\left(r_{0}\right)}{r_{0}^{N-1}}
$$

we can combine Eqs. (27) and (28) to express $S$ as

$$
S=-\mu_{0} \frac{r_{a}}{r^{N}}\left[J r_{a} X_{a, J}^{(N)}(r)+M_{\mathrm{sat}} X_{a, M}^{(n)}(r)\right] .
$$

Finally, using Eqs. (7), (12) and (23), we find

$$
S=\frac{-\mu_{0}}{\left(r_{a}+w\right)^{N-2}}\left[J .\left(X_{J}+A_{\mu} X_{J, s}\right)+\frac{M_{\mathrm{sat}}}{r_{a}+w} \cdot\left(X_{M}+A_{\mu} X_{M, s}\right)\right] .
$$

This formula applies for the magnet strength of a sector coil with $2 \mathrm{~N}$ poles, an iron screen and saturated iron poles, with aperture $r_{a}$, coil width $w$, coil to iron screen gap $g$ (as defined in Fig. 1), engineering current density $J$ and saturation magnetization of the iron poles $M_{\text {sat }}\left(M_{\text {sat }}=0\right.$ if no iron poles, $M_{\text {sat }}=1.7 \times 10^{6} \mathrm{~A} / \mathrm{m}$ for standard iron).

Moreover, $A_{\mu}$ is a correction factor for the iron screen contribution given by

$$
A_{\mu}=a_{\mu}\left(\frac{r_{a}+w}{r_{s}}\right)^{2 N},
$$

where $r_{s}=r_{a}+w+g$. The parameter $a_{\mu}$ given in Eq. (4) is a coefficient linked to the iron screen saturation and thus depends on the relative magnetic permeability $\mu_{r}$ of the iron screen. Its value is bounded between 0 and 1 , the two extreme cases corresponding to the absence of iron screen 
(for which $\mu_{r}=1$ and thus $a_{\mu}=0$ ) and to an unsaturated iron screen (for which $\mu_{r} \gg 1$ and thus $a_{\mu}=1$, which is the case considered in [4]). The exact value of $a_{\mu}$ cannot be determined analytically but its average from 0 to $4 \mathrm{~T}$ (typical range of magnetic field in iron screen for corrector magnets) leads to a good approximation of its effect in real magnet designs; this average gives $a_{\mu} \simeq 0.8$ from the iron B-H curve used in ROXIE.

In addition, $X_{J}, X_{J, s}, X_{M}$ and $X_{M, s}$ are dimensionless coefficients given by

$$
\begin{aligned}
X_{J} & =\frac{\sqrt{3}}{\pi(N-2)}\left[\left(1+\frac{w}{r_{a}}\right)^{N-2}-1\right], \\
X_{J, s} & =\frac{\sqrt{3}}{\pi(N+2)}\left[1-\left(1+\frac{w}{r_{a}}\right)^{-N-2}\right], \\
X_{M} & =\frac{N}{\pi(N-1)}\left[\left(1+\frac{w}{r_{a}}\right)^{N-1}-1\right], \\
X_{M, s} & =\frac{N}{\pi(N+1)}\left[1-\left(1+\frac{w}{r_{a}}\right)^{-N-1}\right] .
\end{aligned}
$$

$X_{J}$ accounts for the magnetic contribution of the coil current, $X_{J, S}$ for its image by the iron screen, $X_{M}$ for the saturated iron poles and $X_{M, s}$ for their image by the iron screen. If $N=2$ (i.e., for the quadrupole), $X_{J}$ must be replaced by

$$
X_{J}=\frac{\sqrt{3}}{\pi} \ln \left(1+\frac{w}{r_{a}}\right)
$$

If $N=1$ (i.e., for the dipole), $X_{M}$ must be replaced by

$$
X_{M}=\frac{1}{\pi} \ln \left(1+\frac{w}{r_{a}}\right)
$$

Furthermore, for comparison purposes we have derived the magnet strength from the magnetic field expressions given by Asner in [4] for the cases he explored (i.e., from the dipole up to the octupole). It has then appeared that Eq. (30) with $a_{\mu}=1$ and $M_{\text {sat }}=0$ (i.e., unsaturated iron screen and no iron poles) is in exact analytical agreement with the strength derived from Asner's formulas. Consequently, for the case without iron poles the difference between the strengths depends on the iron screen saturation and thus on the value of $a_{\mu}$ : as an example, not considering the saturation (i.e., $a_{\mu}=1$ ) would lead to a discrepancy that would not necessarily be negligible but that would

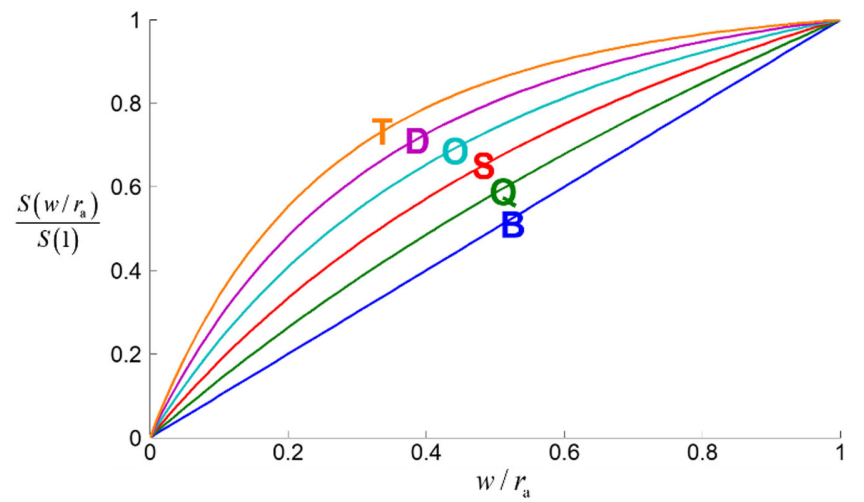

FIG. 3. Normalized strength versus $w / r_{a}$ for sector coils without iron screen with different number of poles (BQSODT: from dipole to dodecapole).

nonetheless not exceed $25 \%$ compared to the taking into account of the saturation (i.e., $a_{\mu} \simeq 0.8$, typical average value discussed above). In addition, in [8] we have shown that the relative difference between the magnet strength computed analytically with Eq. (30) and its numerical evaluation with Roxie on cases representative of the LHC lattice sextupole (MS) and the FCC lattice octupole (MO) is less than $3 \%$, even in the presence of iron poles.

Finally, note that, unlike dipoles, the magnet strength does not scale linearly with the coil width $w$ for quadrupoles and higher order magnets, as shown in Fig. 3. However, under the assumptions that the coil width is negligible with respect to the aperture radius and that the coil to iron screen gap is zero, i.e., $w / r_{a} \ll 1$ and $g=0$, using a first-order Taylor series approximation on Eq. (30), the magnet strength becomes close to

$$
S \simeq-\frac{\mu_{0}}{\pi} \frac{w}{r_{a}{ }^{N-1}}\left[J \sqrt{3}+N \frac{M_{\mathrm{sat}}}{r_{a}}\right]\left[1+a_{\mu}\right]
$$

\section{B. Peak field on conductor}

From the expressions of the magnetic vector potential in the sector coil winding $A_{z}^{(b, t o t)}$ given by Eqs. (13) and (24) and using $\vec{B}=\vec{\nabla} \times \vec{A}$, we have been able to express the magnetic field in the sector coil winding as a finite sum (see the Appendix) using a methodology inspired by [9]. This has enabled us to express the peak field on conductor $B_{p}$ in a sector coil as

$$
B_{p}=\max _{r}\left\{\mu_{0} \sqrt{\left[J r_{a} b_{r}^{(J)}(r)+M_{\mathrm{sat}} b_{r}^{(M)}(r)\right]^{2}+\left[J r_{a} b_{\theta}^{(J)}(r)+M_{\mathrm{sat}} b_{\theta}^{(M)}(r)\right]^{2}}\right\}
$$

where $r \in\left[r_{a}+w_{i} ; r_{a}+w-w_{i}\right]$ with $w_{i}$ the radial insulation thickness of the conductor and with 


$$
\begin{aligned}
& b_{r}^{(J \text { or } M)}(r)=-\sum_{k=0}^{2 N-1}(-1)^{k} \operatorname{Im}\left[U_{J \text { or } M}^{(k)}(r)+V_{J \text { or } M}^{(k)}(r)+W_{J \text { or } M}^{(k)}(r)\right] \\
& b_{\theta}^{(J \text { or } M)}(r)=-\sum_{k=0}^{2 N-1}(-1)^{k} \operatorname{Re}\left[-U_{J \text { or } M}^{(k)}(r)+V_{J \text { or } M}^{(k)}(r)+W_{J \text { or } M}^{(k)}(r)\right],
\end{aligned}
$$

where $\operatorname{Re}(x)$ and $\operatorname{Im}(x)$ are the real and imaginary parts of $x$ respectively, and $U_{J}^{(k)}(r), V_{J}^{(k)}(r), W_{J}^{(k)}(r)$ are dimensionless complex functions of $r$ given by

$$
\begin{aligned}
U_{J}^{(k)}(r) & =\frac{1}{2 i \pi} \frac{r}{r_{a}}\left[f_{J}\left(\frac{r_{a}}{r}, 1, a_{k}^{-}\right)-f_{J}\left(\frac{r_{a}}{r}, 1, a_{k}^{+}\right)\right] \\
V_{J}^{(k)}(r) & =\frac{1}{2 i \pi} \frac{r}{r_{a}}\left[g_{J}\left(\frac{r}{r_{a}+w}, 1, a_{k}^{-}\right)-g_{J}\left(\frac{r}{r_{a}+w}, 1, a_{k}^{+}\right)\right] \\
W_{J}^{(k)}(r) & =a_{\mu}\left(\frac{r_{s}}{r}\right)^{4} \frac{1}{2 i \pi} \frac{r}{r_{a}}\left[f_{J}\left(\frac{r_{a} r}{r_{s}^{2}}, \frac{\left(r_{a}+w\right) r}{r_{s}^{2}}, a_{k}^{-}\right)-f_{J}\left(\frac{r_{a} r}{r_{s}^{2}}, \frac{\left(r_{a}+w\right) r}{r_{s}^{2}}, a_{k}^{+}\right)\right]
\end{aligned}
$$

and $U_{M}^{(k)}(r), V_{M}^{(k)}(r), W_{M}^{(k)}(r)$ are dimensionless complex functions of $r$ given by

$$
\begin{aligned}
U_{M}^{(k)}(r)= & \frac{1}{4 \pi}\left[-f_{M}\left(\frac{r_{a}}{r}, 1, a_{k}^{-}\right)-f_{M}\left(\frac{r_{a}}{r}, 1, a_{k}^{+}\right)+f_{M}\left(\frac{r_{a}}{r}, 1, a_{k}^{2-}\right)+f_{M}\left(\frac{r_{a}}{r}, 1, a_{k}^{2+}\right)\right] \\
V_{M}^{(k)}(r)= & \frac{1}{4 \pi}\left[-g_{M}\left(\frac{r}{r_{a}+w}, 1, a_{k}^{-}\right)-g_{M}\left(\frac{r}{r_{a}+w}, 1, a_{k}^{+}\right)+g_{M}\left(\frac{r}{r_{a}+w}, 1, a_{k}^{2-}\right)+g_{M}\left(\frac{r}{r_{a}+w}, 1, a_{k}^{2+}\right)\right] \\
W_{M}^{(k)}(r)= & a_{\mu}\left(\frac{r_{s}}{r}\right)^{2} \frac{1}{4 \pi}\left[-f_{M}\left(\frac{r_{a} r}{r_{s}{ }^{2}}, \frac{\left(r_{a}+w\right) r}{r_{s}^{2}}, a_{k}^{-}\right)-f_{M}\left(\frac{r_{a} r}{r_{s}^{2}}, \frac{\left(r_{a}+w\right) r}{r_{s}^{2}}, a_{k}^{+}\right)\right. \\
& \left.+f_{M}\left(\frac{r_{a} r}{r_{s}{ }^{2}}, \frac{\left(r_{a}+w\right) r}{r_{s}{ }^{2}}, a_{k}^{2-}\right)+f_{M}\left(\frac{r_{a} r}{r_{s}{ }^{2}}, \frac{\left(r_{a}+w\right) r}{r_{s}{ }^{2}}, a_{k}^{2+}\right)\right],
\end{aligned}
$$

where $i$ is the imaginary unit and where

$$
a_{k}^{ \pm}=e^{i\left(\theta_{0}+k \pi / N \pm \alpha_{N}\right)}, \quad a_{k}^{2 \pm}=e^{i\left(\theta_{0}+k \pi / N \pm 2 \alpha_{N}\right)}
$$

and

$$
\begin{aligned}
f_{J}\left(\rho_{1}, \rho_{2}, a\right) & =\frac{1}{4 a^{2}}\left[2\left(a^{2} \rho^{2}-1\right) \ln (1-a \rho)-a \rho(a \rho+2)\right]_{\rho_{1}}^{\rho_{2}} \\
g_{J}\left(\rho_{1}, \rho_{2}, a\right) & =\frac{1}{2}\left[\frac{a^{2} \rho^{2}-1}{\rho^{2}} \ln (1-a \rho)-a^{2} \ln (\rho)+\frac{a}{\rho}\right]_{\rho_{1}}^{\rho_{2}}
\end{aligned}
$$

and

$$
\begin{aligned}
& f_{M}\left(\rho_{1}, \rho_{2}, a\right)=\left[\frac{1}{a} \ln (1-a \rho)+\rho\right]_{\rho_{1}}^{\rho_{2}} \\
& g_{M}\left(\rho_{1}, \rho_{2}, a\right)=a[\ln (1-a \rho)-\ln (\rho)]_{\rho_{1}}^{\rho_{2}}
\end{aligned}
$$

where $[f(\rho)]_{\rho_{1}}^{\rho_{2}}=f\left(\rho_{2}\right)-f\left(\rho_{1}\right), \theta_{0}=\alpha_{N}-\arctan \left(h_{i} / r_{a}\right)$, with $h_{i}$ the azimuthal insulation thickness of the conductor.
From experience, the peak field is always located on the side of the winding in the azimuthal direction, i.e., on the $\theta=\alpha_{N}$ line (see Fig. 1) and at different values of $r$ depending on the number of magnet poles and on the presence of iron poles. However, the above logarithmic formulas $f_{M}$ and $g_{M}$ diverge in the corners of the iron pole, i.e., for the value $\theta=\alpha_{N}$ and $r=r_{a}$ or $r=r_{a}+w$; this comes from singularities of the equivalent current distribution $\overrightarrow{J^{M}}=\vec{\nabla} \times \vec{M}$ in the corners of the iron poles. To avoid evaluating the peak field in these points, we compute it inside the bare conductor and not inside the insulation, i.e., slightly before the iron pole in the azimuthal direction at $\theta_{0}=\alpha_{N}-\arctan \left(h_{i} / r_{a}\right)$ and slightly after the aperture radius at $r=r_{a}+w_{i}$; hence the use of the radial and azimuthal insulation thicknesses $w_{i}$ and $h_{i}$ in the peak field formulas above.

In [8] we have shown that the relative difference between the peak field on conductor computed analytically with Eq. (34) and its numerical evaluation with Roxie on cases representative of the LHC lattice sextupole (MS) and the FCC lattice octupole (MO) is less than 5\%, even in presence of iron poles. 


\section{DESIGN OF COSINE-THETA CORRECTOR MAGNETS: DASH ALGORITHM}

In this section, we will discuss the main assumptions and guidelines we use in DASH to design cosine-theta corrector magnets.

\section{A. Equivalent sector coil}

As mentioned previously, the geometry of cosine-theta coils is similar to that of sector coils for sextupoles and higher order magnets as each sector does not need to be split in different blocks for the optimization of its field quality (conversely to dipoles). Similarly to the approach presented in [10], we thus approximate the strength and peak field on the conductor of a cosine-theta coil using its equivalent sector coil and the formulas derived in Sec. II.

To define the equivalent sector coil (see Fig. 4), we adjust its coil width $w$ such that its area is equal to that of the cosine-theta coil, as in [10], considering that every other parameter is the same (e.g., same engineering current density $J$, same ampere-turn value per coil, same aperture radius $r_{a}$, same iron screen radius $r_{s}$ ). For a cosine-theta coil made of $N_{C}$ Ribbon cables [11], each cable having a height $h_{C}$ and a width $w_{C}$ (as depicted in Fig. 4), the coil width $w$ of its equivalent sector coil can be derived from the equality of coil areas, as in [10], and is given by

$$
w=r_{a}\left[\sqrt{1+\frac{2 w_{C} N_{C} h_{C}}{\alpha_{N} r_{a}^{2}}}-1\right],
$$

where $\alpha_{N}=\pi /(3 N)$ and $2 N$ is the number of poles.

Here we have considered the use of Ribbon cables, but this conversion method can be applied to cosine-theta coils with any other type of conductor (e.g., Rutherford cables);

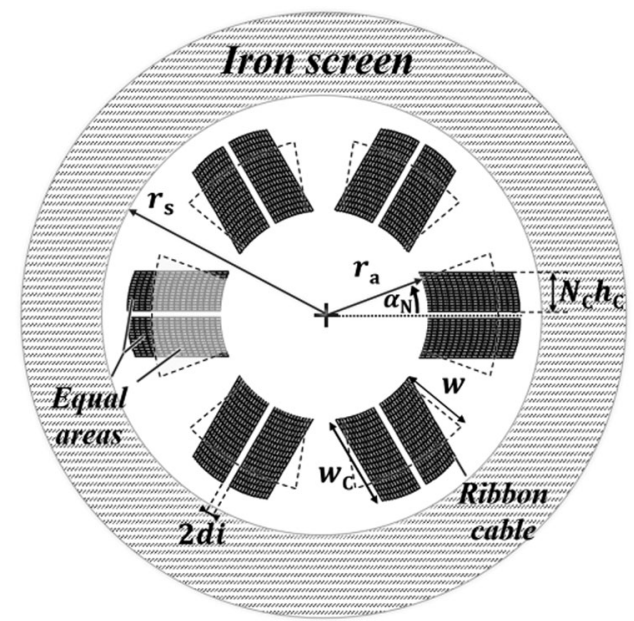

FIG. 4. Sketch of a $2 \mathrm{~N}$-poles cosine-theta magnet with iron screen and its equivalent sector coil (dashed lines) for $N=3$ (i.e., sextupole).
Eq. (3) just needs to be updated with the relevant conductor parameters starting back from the equality of coil areas.

\section{B. Cancellation of the first nonfundamental harmonic}

During the design phase of a cosine-theta magnet, another important point is to control the magnetic field quality. For magnets with $2 N$-poles and $N \geq 3$, this usually just requires to cancel the first nonfundamental harmonic $b_{3 N}$ as the second one $b_{5 N}$ will in practice already be negligible. However, for cosine-theta coils there is no straightforward condition on the winding to fulfill this requirement, conversely to sector coils for which $\alpha_{N}=$ $\pi /(3 N)$ is sufficient. For a given width of Ribbon cable $w_{C}$, it is nevertheless possible to determine the height $N_{C} h_{C}$ of the cosine-theta half-coil (see Fig. 4) such that $b_{3 N}$ is almost zero. Indeed, from the magnetic field expressions we have derived in [8], this can be achieved through the numerical resolution for $N_{C} h_{C}$ of the following equation:

$\int_{r=r_{a}}^{r_{a}+w_{C}}\left\{\sin \left[3 N \theta_{2}(r)\right]-\sin \left[3 N \theta_{1}(r)\right]\right\}\left(\frac{r_{a}}{r}\right)^{3 N-1} d r=0$

with $\theta_{1}(r)=\arcsin \left(\frac{d_{i}}{r}\right)$ and $\theta_{2}(r)=\arcsin \left(\frac{N_{C} h_{C}+d_{i}}{r}\right)$, where $d_{i}$ is half the insulation thickness between one coil and the adjacent one (see Fig. 4).

\section{Load line and quench protection}

In addition, to generate a magnet design which can be operated in the shadow of the dipole magnets, we define a percentage $l$ at which the magnet should be operated along its load line and limit the copper current density to $J_{\mathrm{Cu} \text {,max }}$ such that the magnet can be safely discharged without individual extraction resistor in case of quench (typically $J_{\mathrm{Cu}, \max }=1000 \mathrm{~A} / \mathrm{mm}^{2}$ ). These design requirements correspond to the following system of equations

$$
\begin{aligned}
& J=\frac{l f}{1+\lambda} J_{c}\left[B_{p}(J / l)\right] \\
& J=\frac{f \lambda}{1+\lambda} J_{\mathrm{Cu}, \max },
\end{aligned}
$$

where $f$ is the filling factor of the coil (i.e., copper and superconductor area over total coil area including insulation), $\lambda$ is the copper to noncopper ratio of the conductor. $J_{c}(B)$ is the fit of critical current density of the superconductor and $B_{p}(J)$ is the peak field on conductor whose formula has been given in Sec. III B. The resolution of this system can be achieved through the numerical solving for the operating engineering current density $J_{\text {op }}$ of the following equation:

$$
\left(f-J_{\mathrm{op}} / J_{\mathrm{Cu}, \max }\right) l J_{c}\left[B_{p}\left(J_{\mathrm{op}} / l\right)\right]-J_{\mathrm{op}}=0
$$


and by using the following formula to deduce the copper to noncopper ratio $\lambda$ :

$$
\lambda=J_{\mathrm{op}} /\left(f J_{\mathrm{Cu}, \max }-J_{\mathrm{op}}\right) .
$$

\section{Magnet cost and complexity reduction}

It shall be noted that for high-energy circular colliders equipped with high field dipole magnets, an overall cost optimization calls for covering as much as possible the collider circumference with these magnets. By this approach the highest integrated field and energy can be obtained with the lowest magnetic field in the dipoles (and associated cost). Following this reasoning, to reach the integrated strengths required by the beam dynamics, the corrector magnets shall then be short and shall thus have high magnetic strengths. However, as explained in [12], it is not recommended to select the highest magnetic strength possible for corrector magnets as the save in their length will eventually become marginal compared to the increase in their cost and complexity. Consequently, a reasonable compromise has to be found between corrector magnets length, cost and complexity; we have presented in [12] a first order optimization method with respect to this problem.

In this approach that we will not detail here, we considered that the efficiency of a corrector magnet with respect to cost and complexity was increasing with decreasing ratio $V_{\mathrm{sc}} / S_{\text {int }}$ of superconductor volume over integrated strength, which is equivalent to the ratio $A_{\mathrm{sc}} / S$ of superconductor area over magnet strength, given by

$$
\frac{V_{\mathrm{sc}}}{S_{\mathrm{int}}}=\frac{A_{\mathrm{sc}}}{S}=4 N \frac{f}{1+\lambda} \frac{N_{C} h_{C} w_{C}}{S}
$$

for a Ribbon cable magnet.

\section{E. DASH design methodology}

As mentioned in the Introduction, the purpose of DASH is to automatically design reliable and cost efficient superconducting cosine-theta corrector magnets. To fulfill these requirements, we have implemented the following methodology.

(i) DASH user inputs correspond to the aperture radius $r_{a}$, the order of the corrector magnet $N$, the operating temperature, the presence of iron screen and poles and the percentage along the load line $l$ at which the magnet will be operated. A required strength $S_{\text {req }}$ can also be specified by the user.

(ii) DASH generates a loop over the cosine-theta coil width $w_{C}$ in which: (i) it numerically solves Eq. (4) to determine the height $N_{C} h_{C}$ of the cosine-theta half coil such that its first nonfundamental harmonic $b_{3 N}$ is almost zero; (ii) it deduces the width $w$ of the equivalent sector coil from the values of $w_{C}$ and $N_{C} h_{C}$ and Eq. (3); (iii) from the
$\mathrm{Nb}$-Ti fit of the critical current density $J_{c}$ used in ROXIE [13] and the peak field formula given by Eq. (2), it defines and numerically solves Eq. (5) to determine the operating engineering current density $J_{\text {op }}$ and corresponding magnet strength $S_{\text {op }}$, and it deduces the copper to noncopper ratio $\lambda$ using Eq. (6).

(iii) From this parametric study, it selects the optimal cosine-theta coil width $w_{C}{ }^{*}$ that allows to either reach the required strength $S_{\text {req }}$ with the minimum superconductor volume or to obtain a reasonable compromise between magnet length, cost and complexity (following the method described in [12]).

(iv) From the parameters associated with the optimal cosine-theta coil width $w_{C}{ }^{*}$ (e.g., corresponding $N_{C} h_{C}{ }^{*}$, $J_{\mathrm{op}}{ }^{*}, \lambda^{*}$, etc.), it defines the real conductor parameters (e.g., nominal current, strand dimensions, number of strands per cable, etc.) and generates the corresponding 2D ROXIE model for a numerical evaluation of the proposed optimal design. DASH also computes a reliable estimate of the inductance and stored energy per unit length of the magnet; these values are useful for first considerations on the quench protection.

In practice, the evaluation of the 2D ROXIE model generated by DASH leads to a good approximation of the requested percentage $l$ along the load line (within 1\%, see [8]). Moreover, if needed DASH can automatically read the ROXIE output file to refine the copper to noncopper ratio $\lambda$ and the current per conductor in order to be closer to $l$. After the refinement, the new percentage along the load line computed by ROXIE is matching the one requested by the DASH user (within $0.01 \%$, see [8]).

\section{APPLICATION OF DASH TO GENERIC STUDIES}

DASH has turned out to be a useful tool during the design phase of the FCC lattice sextupoles (MS), lattice octupoles (MO) and sextupole spool piece corrector magnets (MCS) [12]. This design study has led to consistent results with respect to FCC requirements and has thus established the validity of this algorithm as an automatic design tool.

Furthermore, in order to both illustrate the design abilities of DASH and to provide premade designs and useful considerations on $\mathrm{Nb}$ - $\mathrm{Ti}$ corrector magnets ranging from sextupole to dodecapole to the community, we will present here the DASH designs obtained with the optimization method described in [12] (compromise between magnet length, cost and complexity). In addition, since DASH can also handle the presence of iron poles and nested magnet configurations (using DASH2in1 algorithm [8]), we will present the results of these generic design studies as well.

To define a common baseline between these different design studies, we have set the following realistic 


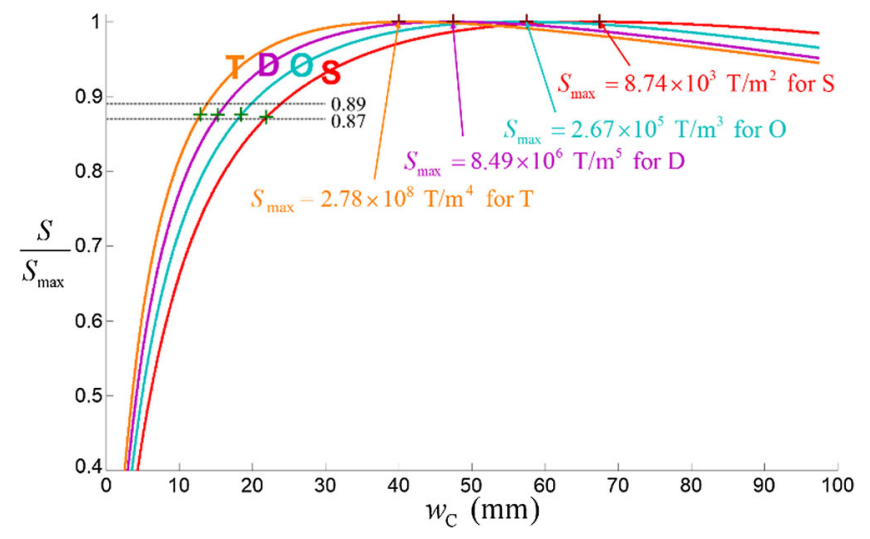

FIG. 5. Strength at $80 \%$ along the magnet load line normalized to its maximum value versus coil width $w_{C}$ for cosine-theta magnets ranging from sextupole to dodecapole (SODT) without iron poles.

parameters: aperture radius $r_{a}=25 \mathrm{~mm}$, percentage along the magnet load line $l=80 \%$, operating temperature $T_{\text {op }}=1.9 \mathrm{~K}$, distance between coil and iron screen $g=$ $3.78 \mathrm{~mm}$ (taken identical to the one of LHC MS, as a conservative reference), maximum copper current density $J_{\mathrm{Cu}, \max }=1000 \mathrm{~A} / \mathrm{mm}^{2}$. To avoid any ambiguity, the term "iron screen" refers to the iron scissor laminations used in the LHC corrector magnets (see [11]).

\section{A. Corrector magnets without iron poles}

The results of the generic design study we have performed on corrector magnets without iron poles are presented through Fig. 5 and Table I. In Fig. 5, we have displayed the strength that a cosine-theta magnet (ranging from sextupole to dodecapole) reaches at $80 \%$ along its load line with a maximum copper current density of $J_{\mathrm{Cu} \text { max }}=1000 \mathrm{~A} / \mathrm{mm}^{2}$ as a function of its coil width $w_{C}$; we have normalized each strength versus width curve to its maximum value (red crosses on the right in Fig. 5). The green crosses on the left in Fig. 5 correspond to the result of the optimization made in DASH as discussed in the introduction of this section; the corresponding optimal design parameters are detailed in Table I. The coil width, number of turns, current per wire, copper to noncopper ratio, wire dimensions and the estimates of the inductance and stored energy per unit magnet length displayed in Table I are directly computed by DASH while the magnet strength, peak field on conductor and magnetic field harmonics are computed using the ROXIE model generated by DASH. Note that the results of the optimization for the sextupole and octupole magnets are slightly different than those presented in [12] for which we chose to slightly decrease their coil width to reduce their inductance.

The magnet parameters presented in Table I can directly be used as premade designs. If the design requirements are different than those presented at the beginning of the section, the parameters of Table I can be used as a starting point; as an indication, for $l<80 \%$, or for $r_{a}>25 \mathrm{~mm}$, the corresponding optimal coil width will be slightly lower.

\section{B. Corrector magnets with iron poles}

We have also performed the same generic design study on corrector magnets with iron poles; the results are presented through Fig. 6 and Table II.

As a summary of this generic design study, the use of iron poles allows to reach higher strengths (by about $15 \%$ for the sextupole, $20 \%$ for the octupole, $25 \%$ for the decapole and $30 \%$ for the dodecapole) for the same coil width (and thus the same superconductor volume) and for the same percentage along the magnet load line. At the same time, their use will inevitably degrade the magnetic field quality in the aperture, as visible in Table II. Nevertheless, this negative impact could be compatible with the beam dynamics requirements of a particle

TABLE I. Optimal parameters obtained with DASH for cosine-theta magnets without iron poles ranging from sextupole to dodecapole. The number of turns and wire size are expressed as "radial $\times$ azimuthal". Harmonics are expressed at reference radius $r_{0}=17 \mathrm{~mm}$.

\begin{tabular}{|c|c|c|c|c|c|}
\hline Magnet order & & $\mathrm{S}(N=3)$ & $\mathrm{O}(N=4)$ & $\mathrm{D}(N=5)$ & $\mathrm{T}(N=6)$ \\
\hline Coil width (mm) & & 21.9 & 18.1 & 15.3 & 13.3 \\
\hline Number of turns & & $18 \times 13$ & $15 \times 9$ & $12 \times 7$ & $11 \times 6$ \\
\hline Current per wire (A) & & 555 & 616 & 678 & 622 \\
\hline $\mathrm{Cu}: \mathrm{NonCu}$ ratio & & 2.6 & 3.5 & 4.2 & 4.9 \\
\hline \multirow[t]{2}{*}{ Wire size $(\mathrm{mm})$} & Bare & $1.16 \times 0.66$ & $1.15 \times 0.69$ & $1.22 \times 0.69$ & $1.15 \times 0.65$ \\
\hline & $\mathrm{Ins}^{\mathrm{a}}$ & $1.22 \times 0.72$ & $1.21 \times 0.75$ & $1.28 \times 0.75$ & $1.21 \times 0.71$ \\
\hline Inductance (mH/m) & & 314 & 122 & 54 & 37 \\
\hline Energy $(\mathrm{kJ} / \mathrm{m})$ & & 48.4 & 23.2 & 12.4 & 7.2 \\
\hline Strength $\left(\mathrm{T} / \mathrm{m}^{N-1}\right)$ & & $7.69 \times 10^{3}$ & $2.30 \times 10^{5}$ & $7.22 \times 10^{6}$ & $2.33 \times 10^{8}$ \\
\hline Peak field $(\mathrm{T})$ & & 5.89 & 4.73 & 3.92 & 3.29 \\
\hline \multirow[t]{2}{*}{ Harmonics $\left(10^{-4}\right.$ units $)$} & $\max$ & $b_{15}<3$ & $b_{20}<1$ & $b_{15}<0.2$ & $b_{18}<0.1$ \\
\hline & Other & $<0.5$ & $<0.2$ & $<0.1$ & $<0.1$ \\
\hline
\end{tabular}

\footnotetext{
${ }^{\mathrm{a}}$ Ins $=$ Insulated.
} 


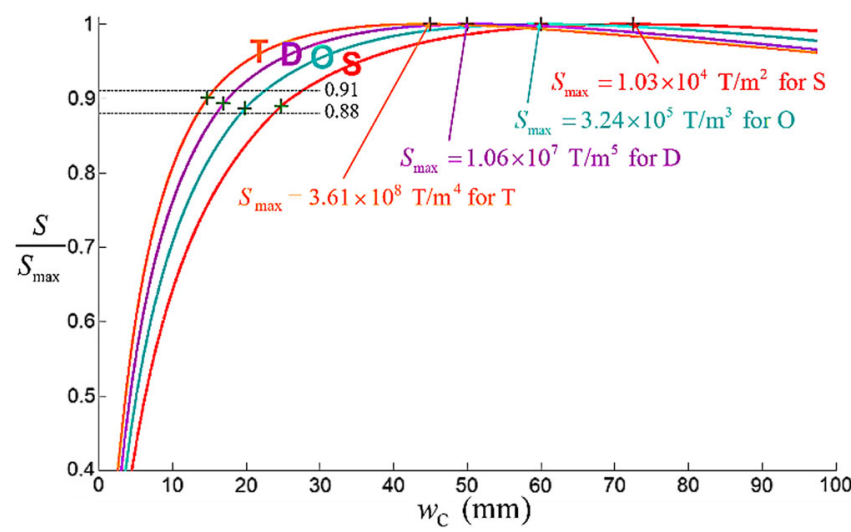

FIG. 6. Strength at $80 \%$ along the magnet load line normalized to its maximum value versus coil width $w_{C}$ for cosine-theta magnets ranging from sextupole to dodecapole (SODT) with iron poles.

accelerator. Indeed, having $b_{9}{ }^{\text {sextupole }}=66$ units at $r_{0}=$ $17 \mathrm{~mm}$ in the aperture along one meter of a sextupole with strength $S_{\text {sextupole }}=9.32 \times 10^{3} \mathrm{~T} / \mathrm{m}^{2}$ is equivalent to having $\quad b_{9}{ }^{\text {dipole }}=b_{9}{ }^{\text {sextupole }} S_{\text {sextupole }} r_{0}^{3-1} / 16 \mathrm{~T}=11$ units along one meter of the 16T FCC-hh main dipole. In addition, taking into account the difference in the total lengths [1] of the FCC-hh main dipoles $(65.8 \mathrm{~km})$ and lattice sextupoles $(0.84 \mathrm{~km})$, the corresponding $b_{9}{ }^{\text {dipole }}$ after rescaling with respect to length would be $b_{9}{ }^{\text {dipole integrated }}=$ $b_{9}{ }^{\text {dipole }} \times 0.84 / 65.8=0.14$ units only.

\section{Nested corrector magnet configurations}

In order to save space in particle accelerators, spoolpiece corrector magnets can be nested as they do not usually have large strength requirements. However, designing such configurations is more complex than for single corrector magnets, as one has to consider the magnetic contribution of the inner magnet on the peak field of the outer magnet and vice versa. Consequently, we have extended the design abilities of DASH to the case of nested corrector magnets through the development of DASH2in1. This algorithm operates with the same method as DASH (see Sec. IV), except that the problem is twodimensional. In addition, it uses the magnetic field formulas we have derived in [8] to evaluate the contribution of one magnet on the peak field of the other.

To illustrate the capabilities of DASH2in1, we will present here a case study for the HE(high energy)-LHC which consists in knowing whether it is possible to nest a sextupole spool piece corrector magnet (MCS) inside a decapole spool piece corrector magnet (MCD) such that they can safely reach strengths of $S_{\mathrm{MCS}}=3 \times 10^{3} \mathrm{~T} / \mathrm{m}^{2}$ and $S_{\mathrm{MCD}}=1.4 \times 10^{6} \mathrm{~T} / \mathrm{m}^{4}$. To carry out this design study, we have used DASH2in1 assuming $r_{a}=25 \mathrm{~mm}$ for the inner magnet and $l=60 \%, T_{\mathrm{op}}=1.9 \mathrm{~K}$, $g=3.78 \mathrm{~mm}, J_{\mathrm{Cu}, \max }=1000 \mathrm{~A} / \mathrm{mm}^{2}$ for both magnets. Moreover, we have set the distance between the inner and outer coils to $g_{c}=1.27 \mathrm{~mm}$, which is the one used in LHC MCDO (MCO inside MCD). In order to optimize the nested magnets cost, DASH2in1 selects the configuration that allows reaching the required strengths with the least total superconductor volume (as discussed in Sec. IV E). The results of this optimization, i.e., the corresponding magnet parameters are displayed in Table III; note that the required strengths are reached with a high level of accuracy.

Moreover, DASH2in1 is also able to compute reliable estimates of the self and mutual inductances per unit length of the nested magnets as well as the corresponding stored energy per unit length using the formulas of the magnetic vector potential given in [8]. From these formulas, it also possible to show-and interesting to remark-that the mutual inductance between nested cosine-theta corrector magnets is always zero for $N_{1} \geq 3$ (number of pairs of poles in the inner magnet) and $N_{2} \geq 3$ (number of pairs of poles in the outer magnet), except if $N_{1}=N_{2}$, which has no use in particle accelerators.

TABLE II. Optimal parameters obtained with DASH for cosine-theta magnets with iron poles ranging from sextupole to dodecapole. The number of turns and wire size are expressed as "radial $\times$ azimuthal." Harmonics are expressed at reference radius $r_{0}=17$ mm.

\begin{tabular}{|c|c|c|c|c|c|}
\hline Magnet order & & $\mathrm{S}(N=3)$ & $\mathrm{O}(N=4)$ & $\mathrm{D}(N=5)$ & $\mathrm{T}(N=6)$ \\
\hline Coil width (mm) & & 24.8 & 19.9 & 17.0 & 14.8 \\
\hline Number of turns & & $20 \times 13$ & $16 \times 9$ & $14 \times 7$ & $12 \times 6$ \\
\hline Current per wire (A) & & 556 & 634 & 644 & 635 \\
\hline $\mathrm{Cu}: \mathrm{NonCu}$ ratio & & 2.4 & 3.4 & 4.3 & 5.0 \\
\hline \multirow[t]{2}{*}{ Wire size $(\mathrm{mm})$} & Bare & $1.18 \times 0.66$ & $1.18 \times 0.69$ & $1.15 \times 0.69$ & $1.17 \times 0.65$ \\
\hline & $\mathrm{Ins}^{\mathrm{a}}$ & $1.24 \times 0.72$ & $1.24 \times 0.75$ & $1.21 \times 0.75$ & $1.23 \times 0.71$ \\
\hline Inductance (mH/m) & & 417 & 154 & 83 & 52 \\
\hline Energy $(\mathrm{kJ} / \mathrm{m})$ & & 64.5 & 30.9 & 17.2 & 10.4 \\
\hline Strength $\left(\mathrm{T} / \mathrm{m}^{N-1}\right)$ & & $9.32 \times 10^{3}$ & $2.92 \times 10^{5}$ & $9.59 \times 10^{6}$ & $3.26 \times 10^{8}$ \\
\hline Peak field $(\mathrm{T})$ & & 5.89 & 4.99 & 4.07 & 3.42 \\
\hline \multirow[t]{2}{*}{ Harmonics $\left(10^{-4}\right.$ units $)$} & $\max$ & $b_{9}<66$ & $b_{12}<38$ & $b_{15}<20$ & $b_{18}<10$ \\
\hline & other & $<0.6$ & $<0.3$ & $<0.1$ & $<0.1$ \\
\hline
\end{tabular}

\footnotetext{
${ }^{\mathrm{a}}$ Ins = Insulated.
} 
TABLE III. Optimal magnet parameters for nested MCS/MCD configuration with respect to required strengths. The number of turns and wire size are expressed as "radial $\times$ azimuthal". Harmonics are expressed at reference radius $r_{0}=17 \mathrm{~mm}$.

\begin{tabular}{|c|c|c|c|}
\hline Magnet type & & Inner magnet $\operatorname{MCS}(N=3)$ & Outer magnet $\operatorname{MCD}(N=5)$ \\
\hline Coil width (mm) & & 4.4 & 2.7 \\
\hline Number of turns & & $3 \times 13$ & $2 \times 9$ \\
\hline Current per wire (A) & & 668 & 643 \\
\hline $\mathrm{Cu}: \mathrm{NonCu}$ ratio & & 3.2 & 3.6 \\
\hline \multirow{2}{*}{ Wire size $(\mathrm{mm})$} & Bare & $1.41 \times 0.62$ & $1.29 \times 0.64$ \\
\hline & $\operatorname{Ins}^{\mathrm{a}}$ & $1.47 \times 0.68$ & $1.35 \times 0.70$ \\
\hline Self inductance $(\mathrm{mH} / \mathrm{m})$ & & 10.4 & 4.0 \\
\hline Mutual inductance $(\mathrm{mH} / \mathrm{m})$ & & 0.0 & 0.0 \\
\hline Energy (kJ/m) & & 2.3 & 0.8 \\
\hline Strength $\left(\mathbf{T} / \mathbf{m}^{N-1}\right)$ & & $3.01 \times 10^{3}$ & $1.40 \times 10^{6}$ \\
\hline Peak field $(\mathrm{T})$ & & 3.25 & 3.15 \\
\hline Harmonics $\left(10^{-4}\right.$ units $)$ & $\max$ & $b_{15}<8.2$ & $b_{15}<0.1$ \\
\hline & Other & $<0.6$ & $<0.1$ \\
\hline
\end{tabular}

${ }^{\mathrm{a}}$ Ins $=$ Insulated.

\section{CONCLUSION}

We have developed new analytical formulas for sector coils. Compared to the already existing formulas that can be found in the literature [4,7], the innovation lies in the generalization to sector coils with any number of poles, the consideration of the iron screen saturation and of the magnetic effect of saturated iron poles. In [8], we have cross-checked these formulas with previous ones [4] and with ROXIE results, and have thus established their validity.

Furthermore, thanks to these analytical formulas and to analogies we have made between sector coils and cosinetheta coils (similar to the ones considered in $[10,14]$ ), we have been able to develop DASH and DASH2in1, which can reliably and rapidly design single or nested cosine-theta magnets with at least six poles and can automatically generate the corresponding 2D ROXIE models. Throughout this development, we have been able to propose methods to rationalize the design of corrector cosine-theta magnets. Moreover, the broad range of comparisons with ROXIE we have presented in [8] has established the reliability of these tools which have been used to design the FCC sextupole and octupole magnets [12].

As a summary, in this paper the reader can find practical and accurate analytical expressions of the magnet strength and peak field on conductor as well as the explicit steps of the algorithm we have defined for the design of corrector magnets designs.

With these new tools in hand, we have been able to carry out generic design studies whose results can be directly used as premade designs or can be considered as reliable starting points for designs with different requirements. They have also shown that the use of iron poles allows reaching higher strengths for a similar cost (i.e., same superconductor volume) and stability (i.e., same percentage along magnet load line) and that their negative impact on the magnetic field quality can still be compatible with beam dynamics requirements of particle accelerators. These generic studies have also shown that DASH and DASH2in1 can automatically design magnets with a good trade-off along their cost and length or to optimize their cost with respect to specific strength requirements in single or nested magnet configurations. These algorithms could be improved in the future to take into account additional design constraints such as mechanical limits or quench simulations.

\section{APPENDIX: DERIVATION OF PEAK FIELD FORMULAS}

In this Appendix, we will provide the methodology (inspired by [9]) to compute the analytical expressions of the peak field on conductor $B_{p}$ presented in Sec. III B through Eqs. (34) to (40). In order to do so, we first need to derive the magnetic field in the sector coil winding, which can be expressed either as an infinite series-as for the associated vector potential $A_{z}^{(b, \text { tot })}$ given in Eqs. (13) and (24) — or as a finite sum of complex functions, i.e., as in Eqs. (34) to (40). Both formulations can be used since they are equivalent and can be evaluated numerically. The main difference lies in the required computation time, which is lower using the finite sum of complex functions; this is the reason why we have chosen to present this formulation here.

\section{Series associated with the peak field for the case without iron poles}

In Sec. II A, we have derived the expression of the magnetic vector potential in the winding of a sector coil; this expression is recalled here: 


$$
\begin{aligned}
& A_{z}^{(b, \text { tot })}(r, \theta) \\
& =\mu_{0} J r_{a}{ }^{2} \sum_{n=1}^{\infty} \frac{\left[X_{b, J}^{(n)}(r)+Y_{b, J}^{(n)}(r)+Z_{b, J}^{(n)}(r)\right] \cos (n \theta)}{n}
\end{aligned}
$$

with $X_{b, J}^{(n)}(r), Y_{b, J}^{(n)}(r), Z_{b, J}^{(n)}(r)$ dimensionless functions of $r$ given in Eq. (14). For our purposes, we have chosen to derive the following alternative expression of these functions:

$$
\begin{aligned}
X_{b, J}^{(n)}(r)= & \frac{\sin \left(n \alpha_{N}\right)}{\pi n} \sum_{k=0}^{2 N-1} e^{i k \pi\left(1+\frac{n}{N}\right)} r_{a}{ }^{-2} r^{-n} \int_{r^{\prime}=r_{a}}^{r} r^{\prime n+1} d r^{\prime} \\
Y_{b, J}(n)(r)= & \frac{\sin \left(n \alpha_{N}\right)}{\pi n} \sum_{k=0}^{2 N-1} e^{i k \pi\left(1+\frac{n}{N}\right)} r_{a}{ }^{-2} r^{n} \int_{r^{\prime}=r}^{r_{a}+w} r^{\prime 1-n} d r^{\prime} \\
Z_{b, J}^{(n)}(r)= & \frac{\sin \left(n \alpha_{N}\right)}{\pi n} \sum_{k=0}^{2 N-1} e^{i k \pi\left(1+\frac{n}{N}\right)} r_{a}{ }^{-2} a_{\mu} r_{a}^{-2 n} r^{n} \\
& \times \int_{r^{\prime}=r_{a}}^{r_{a}+w} r^{\prime n+1} d r^{\prime} .
\end{aligned}
$$

Note that this alternative expression is simply obtained by not solving the integrals on $r_{\vec{A}}^{\prime}$ present in Eq. (45).

Then, using the equation $\vec{B}=\vec{\nabla} \times \vec{A}$, we can express the magnetic field components in the sector coil winding as

$$
\begin{aligned}
& B_{r}^{(b, \mathrm{tot})}(r, \theta)=\frac{1}{r} \frac{\partial A_{z}^{(b, \mathrm{tot})}}{\partial \theta}(r, \theta) \\
& B_{\theta}^{(b, \mathrm{tot})}(r, \theta)=-\frac{\partial A_{z}^{(b, \mathrm{tot})}}{\partial r}(r, \theta)
\end{aligned}
$$

which, using Eq. (A1), leads to

$$
\begin{aligned}
& B_{r}^{(b, \text { tot })}(r, \theta) \\
& =-\mu_{0} J r_{a}^{2} \sum_{n=1}^{\infty} \frac{\left[X_{b, J}^{(n)}(r)+Y_{b, J}{ }^{(n)}(r)+Z_{b, J}^{(n)}(r)\right] \sin (n \theta)}{r} \\
& B_{\theta}^{(b, \text { tot })}(r, \theta) \\
& =-\mu_{0} J r_{a}^{2} \sum_{n=1}^{\infty} \frac{\partial\left[X_{b, J}^{(n)}(r)+Y_{b, J}{ }^{(n)}(r)+Z_{b, J}^{(n)}(r)\right]}{\partial r} \frac{\cos (n \theta)}{n} .
\end{aligned}
$$

In addition, from the expression of $X_{b, J}^{(n)}(r), Y_{b, J}^{(n)}(r)$, $Z_{b, J}^{(n)}(r)$ given in Eq. (A2), it appears that

$$
\begin{aligned}
& \frac{\partial\left[X_{b, J}^{(n)}(r)+Y_{b, J}^{(n)}(r)+Z_{b, J}^{(n)}(r)\right]}{\partial r} \\
& \quad=\frac{n}{r}\left[-X_{b, J}^{(n)}(r)+Y_{b, J}^{(n)}(r)+Z_{b, J}^{(n)}(r)\right] .
\end{aligned}
$$

From this, we can readily derive

$$
\begin{aligned}
& B_{r}^{(b, \text { tot })}(r, \theta)=-\mu_{0} J r_{a}^{2} \sum_{n=1}^{\infty} \frac{\left[X_{b, J}^{(n)}(r)+Y_{b, J}^{(n)}(r)+Z_{b, J}^{(n)}(r)\right] \sin (n \theta)}{r} \\
& B_{\theta}^{(b, \text { tot })}(r, \theta)=-\mu_{0} J r_{a}^{2} \sum_{n=1}^{\infty} \frac{\left[-X_{b, J}^{(n)}(r)+Y_{b, J}(n)(r)+Z_{b, J}^{(n)}(r)\right] \cos (n \theta)}{r}
\end{aligned}
$$

Replacing $X_{b, J}^{(n)}(r), Y_{b, J}^{(n)}(r), Z_{b, J}^{(n)}(r)$ with their expressions given in Eq. (A2), it is now possible to write $B_{r}^{(b, \text { tot })}(r, \theta)$ and $B_{\theta}^{(b, \text { tot })}(r, \theta)$ as

$$
\begin{aligned}
& B_{r}^{(b, \text { tot })}(r, \theta)=-\mu_{0} J r_{a} \sum_{k=0}^{2 N-1}(-1)^{k} \operatorname{Im}\left[U_{J}^{(k)}(r, \theta)+V_{J}^{(k)}(r, \theta)+W_{J}^{(k)}(r, \theta)\right] \\
& B_{\theta}^{(b, \text { tot })}(r, \theta)=-\mu_{0} J r_{a} \sum_{k=0}^{2 N-1}(-1)^{k} \operatorname{Re}\left[-U_{J}^{(k)}(r, \theta)+V_{J}^{(k)}(r, \theta)+W_{J}^{(k)}(r, \theta)\right],
\end{aligned}
$$

where $\operatorname{Re}(x)$ and $\operatorname{Im}(x)$ are the real and imaginary parts of $x$ respectively, and $U_{J}^{(k)}(r, \theta), V_{J}^{(k)}(r, \theta), W_{J}^{(k)}(r, \theta)$ are dimensionless complex functions of $r$ and $\theta$ given by 


$$
\begin{aligned}
& U_{J}^{(k)}(r, \theta)=\frac{1}{r_{a}} \sum_{n=1}^{\infty} \frac{\sin \left(n \alpha_{N}\right)}{\pi n} e^{i \frac{n k \pi}{N}} e^{i n \theta} r^{-n-1} \int_{r^{\prime}=r_{a}}^{r} r^{\prime n+1} d r^{\prime} \\
& V_{J}^{(k)}(r, \theta)=\frac{1}{r_{a}} \sum_{n=1}^{\infty} \frac{\sin \left(n \alpha_{N}\right)}{\pi n} e^{i \frac{n k \pi}{N}} e^{i n \theta} r^{n-1} \int_{r^{\prime}=r}^{r_{a}+w} r^{\prime 1-n} d r^{\prime} \\
& W_{J}^{(k)}(r, \theta)=\frac{1}{r_{a}} \sum_{n=1}^{\infty} \frac{\sin \left(n \alpha_{N}\right)}{\pi n} e^{i \frac{n k \pi}{N}} e^{i n \theta} a_{\mu} r_{s}^{-2 n} r^{n-1} \int_{r^{\prime}=r_{a}}^{r_{a}+w} r^{\prime n+1} d r^{\prime},
\end{aligned}
$$

where $i$ is the imaginary unit.

Replacing $\sin \left(n \alpha_{N}\right)$ by $\left(e^{i n \alpha_{N}}-e^{-i n \alpha_{N}}\right) /(2 i)$ in the term $U_{J}^{(k)}(r, \theta)$, we can write

$$
\begin{aligned}
& U_{J}^{(k)}(r, \theta) \\
& =\frac{1}{2 i \pi r_{a}} \int_{r^{\prime}=r_{a}}^{r} \frac{r^{\prime}}{r} \sum_{n=1}^{\infty} \frac{1}{n}\left[\left(a_{k}^{+}(\theta) \frac{r^{\prime}}{r}\right)^{n}-\left(a_{k}^{-}(\theta) \frac{r^{\prime}}{r}\right)^{n}\right] d r^{\prime}
\end{aligned}
$$

with

$$
\begin{aligned}
& a_{k}^{+}(\theta)=e^{i\left(\theta+\frac{k \pi}{N}+\alpha_{N}\right)} \\
& a_{k}^{-}(\theta)=e^{i\left(\theta+\frac{k \pi}{N}-\alpha_{N}\right)} .
\end{aligned}
$$

We can then perform the change of variable $\rho=r^{\prime} / r$ in the integral of Eq. (A9) so that

$U_{J}^{(k)}(r, \theta)=\frac{1}{2 i \pi} \frac{r}{r_{a}} \int_{\rho=\frac{r_{a}}{r}}^{1} \rho\left[\sum_{n=1}^{\infty} \frac{\left[a_{k}^{+}(\theta) \rho\right]^{n}}{n}-\sum_{n=1}^{\infty} \frac{\left[a_{k}^{-}(\theta) \rho\right]^{n}}{n}\right] d \rho$.

Since $r_{a} \leq r \leq r_{a}+w$, we can conclude that $0 \leq r_{a} / r \leq$ 1 so that $0 \leq r_{a} / r \leq \rho \leq 1$ inside the integral. Therefore, we can use the following Taylor series:

$$
\sum_{n=1}^{\infty} \frac{(a \rho)^{n}}{n}=-\ln (1-a \rho)
$$

provided that $|a \rho|<1$. This leads to

$$
\begin{aligned}
U_{J}^{(k)}(r, \theta)= & \frac{1}{2 i \pi} \frac{r}{r_{a}}\left[\int_{\rho=\frac{r_{a}}{r}}^{1} \rho \ln \left[1-a_{k}^{-}(\theta) \rho\right] d \rho\right. \\
& \left.-\int_{\rho=\frac{r_{a}}{r}}^{1} \rho \ln \left[1-a_{k}^{+}(\theta) \rho\right] d \rho\right] .
\end{aligned}
$$

Therefore, we obtain the expressions given in Eqs. (36) and (39), i.e.,

$U_{J}^{(k)}(r, \theta)=\frac{1}{2 i \pi} \frac{r}{r_{a}}\left[f_{J}\left(\frac{r_{a}}{r}, 1, a_{k}^{-}(\theta)\right)-f_{J}\left(\frac{r_{a}}{r}, 1, a_{k}^{+}(\theta)\right)\right]$

with

$$
\begin{aligned}
f_{J}\left(\rho_{1}, \rho_{2}, a\right) & =\int_{\rho=\rho_{1}}^{\rho_{2}} \rho \ln (1-a \rho) d \rho \\
& =\frac{1}{4 a^{2}}\left[2\left(a^{2} \rho^{2}-1\right) \ln (1-a \rho)-a \rho(a \rho+2)\right] \rho_{\rho_{1}}^{\rho_{2}},
\end{aligned}
$$

where $[f(\rho)]_{\rho_{1}}^{\rho_{2}}=f\left(\rho_{2}\right)-f\left(\rho_{1}\right)$.

Note that in the integral of the expression of $U_{J}^{(k)}(r, \theta)$ given in Eq. (A11), $\rho$ varies between $r_{a} / r$ and 1. Therefore, given the expressions of $a_{k}^{+}(\theta)$ and $a_{k}^{-}(\theta)$ presented in Eq. (A10), we can have $a_{k}^{+}(\theta)=1$ or $a_{k}^{-}(\theta)=1$ and thus $a_{k}^{+}(\theta) \rho=1$ or $a_{k}^{-}(\theta) \rho=1$ when $\rho=1$, so that the logarithm expression cannot in principle be used. However, we can also write

$$
\int_{\rho=\frac{r_{a}}{r}}^{1} \rho \sum_{n=1}^{\infty} \frac{(a \rho)^{n}}{n} d \rho=\sum_{n=1}^{\infty} \frac{a^{n}}{n}\left[\frac{1-\left(r_{a} / r\right)^{n+2}}{n+2}\right],
$$

where $a=a_{k}^{+}(\theta)$ or $a=a_{k}^{-}(\theta)$. This series converges even when $a=1$ as its general term is dominated by $1 / n^{2}$. So 
this proves that $U_{J}^{(k)}(r, \theta)$ converges even when $a_{k}^{+}(\theta)=1$ or $a_{k}^{-}(\theta)=1$.

At the same time, the function $f_{J}\left(\rho_{1}, \rho_{2}, a\right)$ can be extended to the case where $a \rho_{1}=1$ or $a \rho_{2}=1$ since the term $\left(a^{2} \rho^{2}-1\right) \ln (1-a \rho)$ tends towards zero when $a \rho=$ 1 (well-known reference limit). That is why the form given for $U_{J}^{(k)}(r, \theta)$ by Eqs. (36) and (39) can be used and will converge for any $r$ such that $r_{a} \leq r \leq r_{a}+w$ and any $\theta$.

Nevertheless, in order to evaluate the logarithm term $\ln (1-a \rho)$ numerically when $a \rho=1$, it is recommended to use the form $\ln (1-a \rho+\varepsilon)$ in the expression of $f_{J}\left(\rho_{1}, \rho_{2}, a\right)$ given in Eq. (A15), where $\varepsilon$ should be chosen such that it is negligible compared to 1 , but strictly positive, e.g., $\varepsilon=10^{-10}$.

Applying the same method to the terms $V_{J}^{(k)}(r, \theta)$ and $W_{J}^{(k)}(r, \theta)$ but using the changes of variables $\rho=r / r$ and $\rho=r^{\prime} r / r_{s}^{2}$ respectively in the integrals of Eq. (A8) instead of $\rho=r^{\prime} / r$, we obtain the expressions given in Eqs. (36) and (39), i.e.,

$$
\begin{aligned}
& V_{J}^{(k)}(r, \theta)=\frac{1}{2 i \pi} \frac{r}{r_{a}}\left[g_{J}\left(\frac{r}{r_{a}+w}, 1, a_{k}^{-}(\theta)\right)-g_{J}\left(\frac{r}{r_{a}+w}, 1, a_{k}^{+}(\theta)\right)\right] \\
& W_{J}^{(k)}(r, \theta)=a_{\mu}\left(\frac{r_{s}}{r}\right)^{4} \frac{1}{2 i \pi} \frac{r}{r_{a}}\left[f_{J}\left(\frac{r_{a} r}{r_{s}^{2}}, \frac{\left(r_{a}+w\right) r}{r_{s}^{2}}, a_{k}^{-}(\theta)\right)-f_{J}\left(\frac{r_{a} r}{r_{s}^{2}}, \frac{\left(r_{a}+w\right) r}{r_{s}^{2}}, a_{k}^{+}(\theta)\right)\right],
\end{aligned}
$$

where $f_{J}\left(\rho_{1}, \rho_{2}, a\right)$ is detailed in Eq. (A15) and

$$
g_{J}\left(\rho_{1}, \rho_{2}, a\right)=\int_{\rho=\rho_{1}}^{\rho_{2}} \frac{\ln (1-a \rho)}{\rho^{3}} d \rho=\frac{1}{2}\left[\frac{a^{2} \rho^{2}-1}{\rho^{2}} \ln (1-a \rho)-a^{2} \ln (\rho)+\frac{a}{\rho}\right]_{\rho_{1}}^{\rho_{2}}
$$

These expressions of $V_{J}^{(k)}(r, \theta)$ and $W_{J}^{(k)}(r, \theta)$ also converge for any $r$ such that $r_{a} \leq r \leq r_{a}+w$ and any $\theta$, thus we can conclude that $B_{r}^{(b, \text { tot })}(r, \theta)$ and $B_{\theta}^{(b, \text { tot })}(r, \theta)$ converge for any $r$ such that $r_{a} \leq r \leq r_{a}+w$ and any $\theta$.

\section{Series associated with the peak field for the case with iron poles}

In Sec. II B, we have presented the following expression of the magnetic vector potential in the winding of a sector coil which is due to the saturated iron poles:

$$
A_{z}^{(b, \mathrm{tot})}(r, \theta)=\mu_{0} M_{\mathrm{sat}} r_{a} \sum_{n=1}^{\infty} \frac{\left[X_{b, M}^{(n)}(r)+Y_{b, M}^{(n)}(r)+Z_{b, M}^{(n)}(r)\right] \cos (n \theta)}{n},
$$

where the $X_{b, M}^{(n)}(r), Y_{b, M}^{(n)}(r), Z_{b, M}{ }^{(n)}(r)$ dimensionless functions of $r$ are given in Eq. (25) and whose expressions without solving the integrals present in Eq. (5) are

$$
\begin{gathered}
X_{b, M}(n)(r)=\frac{\sin \left(\frac{n \alpha_{N}}{2}\right) \sin \left(\frac{3 n \alpha_{N}}{2}\right)}{\pi} \sum_{k=0}^{2 N-1} e^{i k \pi\left(1+\frac{n}{N}\right)} r_{a}{ }^{-1} r^{-n} \int_{r^{\prime}=r_{a}}^{r} r^{\prime n} d r^{\prime} \\
Y_{b, M}(n)(r)=\frac{\sin \left(\frac{n \alpha_{N}}{2}\right) \sin \left(\frac{3 n \alpha_{N}}{2}\right)}{\pi} \sum_{k=0}^{2 N-1} e^{i k \pi\left(1+\frac{n}{N}\right)} r_{a}{ }^{-1} r^{n} \int_{r^{\prime}=r}^{r_{a}+w} r^{\prime-n} d r^{\prime} \\
Z_{b, M}{ }^{(n)}(r)=\frac{\sin \left(\frac{n \alpha_{N}}{2}\right) \sin \left(\frac{3 n \alpha_{N}}{2}\right)}{\pi} \sum_{k=0}^{2 N-1} e^{i k \pi\left(1+\frac{n}{N}\right)} r_{a}{ }^{-1} a_{\mu} r_{s}^{-2 n} r^{n} \int_{r^{\prime}=r_{a}}^{r_{a}+w} r^{\prime n} d r^{\prime} .
\end{gathered}
$$


From Eqs. (A3) and (A19), the corresponding magnetic field components are then

$$
\begin{aligned}
& B_{r}^{(b, \text { tot })}(r, \theta)=-\mu_{0} M_{\text {sat }} r_{a} \sum_{n=1}^{\infty} \frac{\left[X_{b, M}{ }^{(n)}(r)+Y_{b, M}{ }^{(n)}(r)+Z_{b, M}{ }^{(n)}(r)\right] \sin (n \theta)}{r} \\
& B_{\theta}^{(b, \text { tot })}(r, \theta)=-\mu_{0} M_{\text {sat }} r_{a} \sum_{n=1}^{\infty} \frac{\partial\left[X_{b, M}{ }^{(n)}(r)+Y_{b, M}{ }^{(n)}(r)+Z_{b, M}{ }^{(n)}(r)\right]}{\partial r} \frac{\cos (n \theta)}{n} .
\end{aligned}
$$

Again, from the expression of $X_{b, M}^{(n)}(r), Y_{b, M}{ }^{(n)}(r), Z_{b, M}{ }^{(n)}(r)$ given in Eq. (A20), it appears that

$$
\frac{\partial\left[X_{b, M}^{(n)}(r)+Y_{b, M}^{(n)}(r)+Z_{b, M}^{(n)}(r)\right]}{\partial r}=\frac{n}{r}\left[-X_{b, M}^{(n)}(r)+Y_{b, M}^{(n)}(r)+Z_{b, M}^{(n)}(r)\right] .
$$

Thus

$$
\begin{aligned}
& B_{r}^{(b, \text { tot })}(r, \theta)=-\mu_{0} M_{\text {sat }} r_{a} \sum_{n=1}^{\infty} \frac{\left[X_{b, M}^{(n)}(r)+Y_{b, M}{ }^{(n)}(r)+Z_{b, M}^{(n)}(r)\right] \sin (n \theta)}{r} \\
& B_{\theta}^{(b, \mathrm{tot})}(r, \theta)=-\mu_{0} M_{\mathrm{sat}} r_{a} \sum_{n=1}^{\infty} \frac{\left[-X_{b, M}^{(n)}(r)+Y_{b, M}{ }^{(n)}(r)+Z_{b, M}^{(n)}(r)\right] \cos (n \theta)}{r}
\end{aligned}
$$

which, replacing $X_{b, M}^{(n)}(r), Y_{b, M}{ }^{(n)}(r), Z_{b, M}^{(n)}(r)$ with their expressions given in Eq. (A20), leads to

$$
\begin{aligned}
& B_{r}^{(b, \text { tot })}(r, \theta)=-\mu_{0} M_{\text {sat }} \sum_{k=0}^{2 N-1}(-1)^{k} \operatorname{Im}\left[U_{M}^{(k)}(r, \theta)+V_{M}^{(k)}(r, \theta)+W_{M}^{(k)}(r, \theta)\right] \\
& B_{\theta}^{(b, \text { tot })}(r, \theta)=-\mu_{0} M_{\text {sat }} \sum_{k=0}^{2 N-1}(-1)^{k} \operatorname{Re}\left[-U_{M}^{(k)}(r, \theta)+V_{M}^{(k)}(r, \theta)+W_{M}^{(k)}(r, \theta)\right],
\end{aligned}
$$

where $U_{M}^{(k)}(r, \theta), V_{M}^{(k)}(r, \theta), W_{M}^{(k)}(r, \theta)$ are dimensionless complex functions of $r$ and $\theta$ given by

$$
\begin{aligned}
& U_{M}^{(k)}(r, \theta)=\sum_{n=1}^{\infty} \frac{\sin \left(\frac{n \alpha_{N}}{2}\right) \sin \left(\frac{3 n \alpha_{N}}{2}\right)}{\pi} e^{i \frac{n k \pi}{N}} e^{i n \theta} r^{-n-1} \int_{r^{\prime}=r_{a}}^{r} r^{\prime n} d r^{\prime} \\
& V_{M}^{(k)}(r, \theta)=\sum_{n=1}^{\infty} \frac{\sin \left(\frac{n \alpha_{N}}{2}\right) \sin \left(\frac{3 n \alpha_{N}}{2}\right)}{\pi} e^{i n k \pi} e^{i n \theta} r^{n-1} \int_{r^{\prime}=r}^{r_{a}+w} r^{\prime-n} d r^{\prime} \\
& W_{M}^{(k)}(r, \theta)=\sum_{n=1}^{\infty} \frac{\sin \left(\frac{n \alpha_{N}}{2}\right) \sin \left(\frac{3 n \alpha_{N}}{2}\right)}{\pi} e^{i \frac{n k \pi}{N}} e^{i n \theta} a_{\mu} r_{s}^{-2 n} r^{n-1} \int_{r^{\prime}=r_{a}}^{r_{a}+w} r^{\prime n} d r^{\prime},
\end{aligned}
$$

where $i$ is the imaginary unit. Replacing the term $\sin \left(\frac{n \alpha_{N}}{2}\right) \sin \left(\frac{3 n \alpha_{N}}{2}\right)$ by $\left(e^{i n \alpha_{N}}+e^{-i n \alpha_{N}}-e^{i 2 n \alpha_{N}}-e^{-i 2 n \alpha_{N}}\right) / 4$ in $U_{M}^{(k)}(r, \theta)$, we can write

$$
U_{M}^{(k)}(r, \theta)=\frac{1}{4 \pi} \int_{r^{\prime}=r_{a}}^{r} \frac{1}{r} \sum_{n=1}^{\infty}\left[\left(a_{k}^{+}(\theta) \frac{r^{\prime}}{r}\right)^{n}+\left(a_{k}^{-}(\theta) \frac{r^{\prime}}{r}\right)^{n}-\left(a_{k}^{2+}(\theta) \frac{r^{\prime}}{r}\right)^{n}-\left(a_{k}^{2-}(\theta) \frac{r^{\prime}}{r}\right)^{n}\right] d r^{\prime},
$$

where $a_{k}^{+}(\theta)$ and $a_{k}^{-}(\theta)$ are given in Eq. (A20) and

$$
\begin{aligned}
& a_{k}^{2+}(\theta)=e^{i\left(\theta+\frac{k \pi}{N}+2 \alpha_{N}\right)} \\
& a_{k}^{2-}(\theta)=e^{i\left(\theta+\frac{k \pi}{N}-2 \alpha_{N}\right)} .
\end{aligned}
$$


Using again the change of variable $\rho=r^{\prime} / r$ in the integral of Eq. (A26) as we did for $U_{J}^{(k)}(r, \theta)$ in Appendix A to transform Eq. (A9) into (A11), we obtain

$$
\begin{aligned}
U_{M}^{(k)}(r, \theta)= & \frac{1}{4 \pi} \int_{\rho=r_{a} / r}^{1}\left[\sum_{n=1}^{\infty}\left[a_{k}^{+}(\theta) \rho\right]^{n}+\sum_{n=1}^{\infty}\left[a_{k}^{-}(\theta) \rho\right]^{n}\right. \\
& \left.-\sum_{n=1}^{\infty}\left[a_{k}^{2+}(\theta) \rho\right]^{n}-\sum_{n=1}^{\infty}\left[a_{k}^{2-}(\theta) \rho\right]^{n}\right] d \rho .
\end{aligned}
$$

This time, we can use the following Taylor series:

$$
\sum_{n=1}^{\infty}(a \rho)^{n}=\frac{a \rho}{1-a \rho}
$$

provided that $|a \rho|<1$, so that

$$
\begin{aligned}
U_{M}^{(k)}(r, \theta)= & \frac{1}{4 \pi}\left[\int_{\rho=r_{a} / r}^{1} \frac{a_{k}^{+}(\theta) \rho}{1-a_{k}^{+}(\theta) \rho} d \rho+\int_{\rho=r_{a} / r}^{1} \frac{a_{k}^{-}(\theta) \rho}{1-a_{k}^{-}(\theta) \rho} d \rho\right. \\
& \left.-\int_{\rho=r_{a} / r}^{1} \frac{a_{k}^{2+}(\theta) \rho}{1-a_{k}^{2+}(\theta) \rho} d \rho-\int_{\rho=r_{a} / r}^{1} \frac{a_{k}^{2-}(\theta) \rho}{1-a_{k}^{2-}(\theta) \rho} d \rho\right] .
\end{aligned}
$$

Therefore, we obtain the expressions given in Eqs. (37) and (40), i.e.,

$$
\begin{aligned}
U_{M}^{(k)}(r, \theta)= & \frac{1}{4 \pi}\left[-f_{M}\left(\frac{r_{a}}{r}, 1, a_{k}^{-}(\theta)\right)-f_{M}\left(\frac{r_{a}}{r}, 1, a_{k}^{+}(\theta)\right)\right. \\
& \left.+f_{M}\left(\frac{r_{a}}{r}, 1, a_{k}^{2-}(\theta)\right)+f_{M}\left(\frac{r_{a}}{r}, 1, a_{k}^{2+}(\theta)\right)\right]
\end{aligned}
$$

with

$$
f_{M}\left(\rho_{1}, \rho_{2}, a\right)=\int_{\rho=\rho_{1}}^{\rho_{2}} \frac{-a \rho}{1-a \rho} d \rho=\left[\frac{1}{a} \ln (1-a \rho)+\rho\right]_{\rho_{1}}^{\rho_{2}},
$$

where $[f(\rho)]_{\rho_{1}}^{\rho_{2}}=f\left(\rho_{2}\right)-f\left(\rho_{1}\right)$.

Again, note that in the integral of the expression of $U_{M}^{(k)}(r, \theta)$ given in Eq. (A28), $\rho$ varies between $r_{a} / r$ and 1. Therefore, given the expressions of $a_{k}^{ \pm}(\theta)$ and $a_{k}^{2 \pm}(\theta)$ presented in Eqs. (A10) and (A27), we can have $a_{k}^{ \pm}(\theta)=1$ or $a_{k}^{2 \pm}(\theta)=1$ and thus $a_{k}^{ \pm}(\theta) \rho=1$ or $a_{k}^{2 \pm}(\theta) \rho=1$ for $\rho=1$, so that the Taylor-series expression (A29) cannot in principle be used. However, we can also write

$$
\int_{\rho=\frac{r_{a}}{r}}^{1} \sum_{n=1}^{\infty}(a \rho)^{n} d \rho=\sum_{n=1}^{\infty} a^{n}\left[\frac{1-\left(r_{a} / r\right)^{n+1}}{n+1}\right]
$$

where $a=a_{k}^{ \pm}(\theta)$ or $a=a_{k}^{2 \pm}(\theta)$, which converges only if $\arg (a) \neq 0$ as its general term is of the form $e^{i n \arg (a)} / n$. So $U_{M}^{(k)}(r, \theta)$ converges only if $a_{k}^{+}(\theta), a_{k}^{-}(\theta), a_{k}^{2+}(\theta), a_{k}^{2-}(\theta)$ are all different from 1. Given the expressions of these coefficients, this implies that $U_{M}^{(k)}(r, \theta)$ converges for any $r$ such that $r_{a} \leq r \leq r_{a}+w$ and any $\theta$ except when $\theta=(6 p \pm$ 1) $\alpha_{N}$ or $\theta=(6 p-3 \pm 1) \alpha_{N}$, with $1 \leq p \leq N$ (note that this actually corresponds to the boundaries between the sector coil and its iron poles, see Fig. 2 and the discussion in Sec. III B).

Finally, applying the same method to the terms $V_{M}^{(k)}(r, \theta)$ and $W_{M}^{(k)}(r, \theta)$ but using the changes of variables $\rho=r / r$ ' and $\rho=r^{\prime} r / r_{s}{ }^{2}$ respectively in the integrals of Eq. (A25) instead of $\rho=r^{\prime} / r$, we obtain the expressions given in Eqs. (37) and (40), i.e.,

$$
\begin{aligned}
V_{M}^{(k)}(r, \theta)= & \frac{1}{4 \pi}\left[-g_{M}\left(\frac{r}{r_{a}+w}, 1, a_{k}^{-}(\theta)\right)-g_{M}\left(\frac{r}{r_{a}+w}, 1, a_{k}^{+}(\theta)\right)+g_{M}\left(\frac{r}{r_{a}+w}, 1, a_{k}^{2-}(\theta)\right)+g_{M}\left(\frac{r}{r_{a}+w}, 1, a_{k}^{2+}(\theta)\right)\right] \\
W_{M}^{(k)}(r, \theta)= & a_{\mu}\left(\frac{r_{s}}{r}\right)^{2} \frac{1}{4 \pi}\left[-f_{M}\left(\frac{r_{a} r}{r_{s}^{2}}, \frac{\left(r_{a}+w\right) r}{r_{s}^{2}}, a_{k}^{-}(\theta)\right)-f_{M}\left(\frac{r_{a} r}{r_{s}^{2}}, \frac{\left(r_{a}+w\right) r}{r_{s}^{2}}, a_{k}^{+}(\theta)\right)\right. \\
& \left.+f_{M}\left(\frac{r_{a} r}{r_{s}{ }^{2}}, \frac{\left(r_{a}+w\right) r}{r_{s}{ }^{2}}, a_{k}^{2-}(\theta)\right)+f_{M}\left(\frac{r_{a} r}{r_{s}{ }^{2}}, \frac{\left(r_{a}+w\right) r}{r_{s}{ }^{2}}, a_{k}^{2+}(\theta)\right)\right]
\end{aligned}
$$

where $f_{M}\left(\rho_{1}, \rho_{2}, a\right)$ is detailed in Eq. (A32) and

$$
g_{M}\left(\rho_{1}, \rho_{2}, a\right)=\int_{\rho=\rho_{1}}^{\rho_{2}} \frac{1}{\rho^{2}} \frac{-a \rho}{1-a \rho} d \rho=a[\ln (1-a \rho)-\ln (\rho)]_{\rho_{1}}^{\rho_{2}} .
$$

These expressions of $V_{M}^{(k)}(r, \theta)$ and $W_{M}^{(k)}(r, \theta)$ also converge for any $r$ such that $r_{a} \leq r \leq r_{a}+w$ and any $\theta$ except when $\theta=(6 p \pm 1) \alpha_{N}$ or $\theta=(6 p-3 \pm 1) \alpha_{N}$, with $1 \leq p \leq N$, and thus so do those of the magnetic field components $B_{r}^{(b, t o t)}(r, \theta)$ and $B_{\theta}^{(b, \text { tot })}(r, \theta)$ in the sector coil winding associated with the saturated iron poles. 
[1] D. Schoerling, Other magnets parameters, at the Fourth Annual Meeting of the Future Circular Collider Study (FCC week), Amsterdam, The Netherlands, 2018, https://indico.cern.ch/event/656491/contributions/2920305/ attachments/1628231/2596868/Schoerling_OtherMagnets_ 2018.pdf.

[2] A. Wolski, Beam Dynamics in High Energy Particle Accelerators (Imperial College Press, London, 2014).

[3] E. Todesco, Magnetic design of superconducting magnets, CERN Yellow Report No. CERN-2014-005, pp. 269-292, 2014, https://doi.org/10.5170/CERN-2014-005.269.

[4] A. Asner, Cylindrical aperture multipoles with constant current density sector windings, CERN Report No. SI/Note MAE/69-15, 1969.

[5] K. H. Mess, P. Schmüser, and S. Wolf, Field calculations, in Superconducting Accelerator Magnets (World Scientific, Singapore, 1996), pp. 45-64.

[6] K. H. Mess and P. Schmüser, Superconducting accelerator magnets, in CAS-CERN Accelerator School: Superconductivity in Particle Accelerators, DESY, Hamburg, Germany, 1988, Report No. CERN-1989-004, pp. 87148, http://cds.cern.ch/record/367115/files/87.pdf.

[7] S. Russenschuck, Field Computation for Accelerator Magnets: Analytical and Numerical Methods for Electromagnetic Design and Optimization (Wiley, Weinheim, Germany, 2010).
[8] A. Louzguiti, Analytical computation of magnetic field inside sector coil, CERN Technical Note TE-MSC, EDMS No. 2037178, 2019, https://edms.cern.ch/file/2037178/1/ Analytical_computation_of_magnetic_field_inside_sector_ coil_v6.docx.

[9] J. P. Boris and A. F. Kuckes, Closed expressions for the magnetic field in two-dimensional multipole configurations, Nucl. Fusion 8, 323 (1968).

[10] L. Rossi and E. Todesco, Electromagnetic design of superconducting dipoles based on sector coils, Phys. Rev. ST Accel. Beams 10, 112401 (2007).

[11] A. Ijspeert et al., Development of a superconducting sextupole-dipole corrector magnet, in 11th International Conference on Magnet Technology (MT-11) (Springer, Dordrecht, 1990), Vol. 1, pp. 200-205, https://doi.org/ 10.1007/978-94-009-0769-0_34.

[12] A. Louzguiti, D. M. Araujo, D. Pracht, M. Prioli, E. Ravaioli, D. Schoerling, and G. Vallone, Optimization of the electromagnetic design of the FCC sextupoles and octupoles, IEEE Trans. Appl. Supercond. 29, 5 (2019).

[13] L. Bottura, A practical fit for the critical surface of NbTi, IEEE Trans. Appl. Supercond. 10, 1 (2000).

[14] L. Rossi and E. Todesco, Electromagnetic design of superconducting quadrupoles, Phys. Rev. ST Accel. Beams 9, 102401 (2006). 